\title{
RIGIDITY AND STABILITY OF EINSTEIN METRICS FOR QUADRATIC CURVATURE FUNCTIONALS
}

\author{
MATTHEW J. GURSKY AND JEFF A. VIACLOVSKY
}

\begin{abstract}
We investigate rigidity and stability properties of critical points of quadratic curvature functionals on the space of Riemannian metrics. We show it is possible to "gauge" the Euler-Lagrange equations, in a self-adjoint fashion, to become elliptic. Fredholm theory may then be used to describe local properties of the moduli space of critical metrics. We show a number of compact examples are infinitesimally rigid, and consequently, are isolated critical points in the space of unit-volume Riemannian metrics. We then give examples of critical metrics which are strict local minimizers (up to diffeomorphism and scaling). A corollary is a local "reverse Bishop's inequality" for such metrics. In particular, any metric $g$ in a $C^{2, \alpha}$-neighborhood of the round metric $\left(S^{n}, g_{S}\right)$ satisfying $\operatorname{Ric}(g) \leq \operatorname{Ric}\left(g_{S}\right)$ has volume $\operatorname{Vol}(g) \geq \operatorname{Vol}\left(g_{S}\right)$, with equality holding if and only if $g$ is isometric to $g_{S}$.
\end{abstract}

\section{Contents}

1. Introduction

2. Local properties of the moduli space

3. Second variation at an Einstein metric

4. Local variational properties of Einstein metrics 33

5. Rigidity for Einstein metrics

6. Stability for critical metrics $\quad 39$

7. Examples

References

\section{INTRODUCTION}

1.1. Quadratic Functionals. Let $M$ be a closed manifold of dimension $n \geq 3$. The total scalar curvature, or Einstein-Hilbert functional, has been deeply studied in Riemannian geometry and we do not attempt to give a survey here. We only remark that Einstein metrics in general are saddle points for the Einstein-Hilbert functional, and rigidity and stability properties of Einstein metrics have been studied in [Koi78, Koi80, Koi82. In this article we are interested in functionals on the space of Riemannian metrics $\mathcal{M}$ which are quadratic in the curvature; see [Bes87, Bla00, Smo07] for surveys. Such functionals have also been widely studied in physics under

The first author has been partially supported under NSF grants DMS-0800084 and DMS-1206661. The second author has been partially supported under NSF grants DMS-0804042 and DMS-1105187. 
the name "fourth-order," "critical," or "quadratic" gravity; see for example [LP11, Mal11, Sch07, Ste78].

Using the standard decomposition of the curvature tensor $R m$ into the Weyl, Ricci and scalar curvature curvature components, a basis for the space of quadratic curvature functionals is

$$
\mathcal{W}=\int|W|^{2} d V, \quad \rho=\int|R i c|^{2} d V, \quad \mathcal{S}=\int R^{2} d V .
$$

In dimension three, $\mathcal{W}$ vanishes, and in dimension four, the Chern-Gauss-Bonnet formula

$$
32 \pi^{2} \chi(M)=\int|W|^{2} d V-2 \int|R i c|^{2} d V+\frac{2}{3} \int R^{2} d V
$$

implies that $\mathcal{W}$ can be written as a linear combination of the other two (plus a topological term). In higher dimensions, the Euler-Lagrange equations of $\mathcal{W}$ have a different structure than the latter two functionals. Consequently, in this paper, we will be interested in the functional

$$
\mathcal{F}_{\tau}[g]=\int|R i c|^{2} d V+\tau \int R^{2} d V
$$

(with $\tau=\infty$ formally corresponding to $\int R^{2} d V$ ) in dimensions $n \geq 3$. In dimensions other than four, the functional $\mathcal{F}_{\tau}$ is not scale-invariant. Therefore, we will consider the volume-normalized functional

$$
\tilde{\mathcal{F}}_{\tau}[g]=\operatorname{Vol}(g)^{\frac{4}{n}-1} \mathcal{F}_{\tau}[g] .
$$

Any Einstein metric is critical for $\tilde{\mathcal{F}}_{\tau}$ (this is not true for $\tilde{\mathcal{W}}$; an additional algebraic condition on the curvature tensor is required).

1.2. Rigidity. As with any geometric variational problem we begin by trying to understand the local properties of the moduli space of critical points. This is the space of metrics $g$ satisfying

$$
\nabla \tilde{\mathcal{F}}_{\tau}[g]=0
$$

modulo diffeomorphism equivalence and scaling, where $\nabla \tilde{\mathcal{F}}_{\tau}$ is the gradient of $\tilde{\mathcal{F}}_{\tau}$. Due to diffeomorphism invariance, the condition (1.4) does not define an elliptic equation in $g$. In order to provide a reasonable description of the moduli space near a critical metric, in Section 2.2 we define a map $P_{g}: \bar{S}_{0}^{2}\left(T^{*} M\right) \rightarrow \bar{S}_{0}^{2}\left(T^{*} M\right)$, where

$$
\bar{S}_{0}^{2}\left(T^{*} M\right)=\left\{h \in S^{2}\left(T^{*} M\right): \int\left(t r_{g} h\right) d V_{g}=0\right\} .
$$

In Section 2.2, we will show that the zeroes of $P_{g}$ correspond exactly to critical metrics in a neighborhood of $g$, up to diffeomorphism and scaling. Furthermore, $P_{g}$ has the advantange of being elliptic. This property guarantees that the kernel of the linearized operator, denoted $H_{\tau}^{1}$, is finite dimensional. Therefore, by using a standard implicit function theorem argument one can define the Kuranishi map $\Psi: H_{\tau}^{1} \rightarrow H_{\tau}^{2}$, for which the moduli space is locally given by the orbit space of the isometry group 
acting on the zero locus $\Psi^{-1}(0)$. Note that here $H_{\tau}^{2}$ denotes the cokernel, but since our linearized operator is self-adjoint this is the same as $H_{\tau}^{1}$ on a compact manifold.

Definition 1.1. Let $M$ be a smooth, closed manifold of dimension $n$, and $g$ a critical

metric for $\tilde{\mathcal{F}}_{\tau}$. The metric $g$ is called infinitesimally rigid (for $\tilde{\mathcal{F}}_{\tau}$ ) if $H_{\tau}^{1}=\{0\}$, and $g$ is called rigid (for $\tilde{\mathcal{F}}_{\tau}$ ) if there exists a $C^{4, \alpha}$ neighborhood $U$ of $g$ in the space of Riemannian metrics such that if $\tilde{g} \in U$ and $\tilde{g}$ satisfies (1.4), then $\tilde{g}=e^{c} \phi^{*} g$ for some $C^{5, \alpha}$-diffeomorphism $\phi: M \rightarrow M$ and a constant $c \in \mathbb{R}$.

A non-zero element $h \in H_{\tau}^{1}$ is called integrable there exists a path $g_{s}$ of critical metrics (for $\tilde{\mathcal{F}}_{t}$ ) with $g_{0}=g$ satisfying $\left.\left(g_{s}\right)^{\prime}\right|_{s=0}=h$. Otherwise, $h$ is called nonintegrable.

In Section 2, we will show that infinitesimal rigidity implies rigidity. Furthermore, it can indeed happen that there are non-trivial elements in $H_{\tau}^{1}$ which are non-integrable. This in fact occurs on $\left(S^{3}, g_{S}\right)$, where $g_{S}$ is the round metric, see Theorem 1.8 below. A similar phenomenon occurs for infinitesimal Einstein deformations on certain Einstein manifolds, the simplest known example being $S^{2} \times \mathbb{C P}^{2 m}$ with $m \geq 2$ [Koi82].

On an Einstein manifold, the Lichnerowicz Laplacian is given by

$$
\Delta_{L} h_{i j}=\Delta h_{i j}+2 R_{i p j q} h^{p q}-\frac{2}{n} R h_{i j}
$$

and $\operatorname{spec}_{T T}\left(-\Delta_{L}\right)$ will denote the set of eigenvalues of $\left(-\Delta_{L}\right)$ restricted to transversetraceless (TT) tensors. Infinitesimal Einstein rigidity is the condition

$$
\frac{2}{n} R \notin \operatorname{spec}_{T T}\left(-\Delta_{L}\right)
$$

see [Bes87, Chapter 12]. In particular, if $g$ fails to be rigid as an Einstein metric then it is not rigid as a critical point of $\tilde{\mathcal{F}}_{\tau}$, but the converse may not be true.

In Section 5 we prove a vanishing result for $H_{\tau}^{1}$ when $g$ is an Einstein metric. The full statement is rather complicated, since it depends on the value of $\tau$ and the dimension $n$; therefore, we only state here the result for the functional $\tilde{\mathcal{F}}_{0}=V$ ol $l^{\frac{4}{n}}-1 \int|R i c|^{2} d V$ (which will be of particular interest for the applications described in Section 1.6):

Theorem 1.2. Let $(M, g)$ be an $n$-dimensional Einstein manifold, $n \geq 3$. When $R>0$, assume $(M, g)$ is not isometric to the round sphere. If

$$
\left\{\frac{2}{n} R, \frac{4}{n} R\right\} \notin \operatorname{spec}_{T T}\left(-\Delta_{L}\right)
$$

then $H_{0}^{1}=\{0\}$ and consequently $g$ is rigid for $\tilde{\mathcal{F}}_{0}$.

The same result holds when $R<0$, assuming $n=3$ or $n=4$.

On the round sphere $\left(S^{n}, g_{S}\right), H_{0}^{1}=\{f g: f \in \Lambda\}$, where $\Lambda$ is the space of first-order spherical harmonics, and these elements are generated by the action of the conformal group. Consequently, $g_{S}$ is in fact rigid, see Section 2.2.

The criterion in Theorem 1.2 only involves the action of the Lichnerowicz Laplacian on transverse-traceless variations of the metric. In fact, one also has to consider 
conformal variations, but rigidity under these variations will follow from the Lichnerowicz estimate for the first eigenvalue of the Laplacian (if $g$ is not isometric to the round sphere), see Section 5. In the negative case there is no a priori lower bound for the spectrum of $(-\Delta)$, as can be seen by considering hyperbolic examples with long "necks". Consequently, in dimensions $n \geq 5$, our rigidity result for negative Einstein metrics require an additional assumption on $\lambda_{1}(-\Delta)$; see Lemma 5.1 and Theorem 5.5 for precise statements.

1.3. Stability and local minimization. We will also consider local minimizing properties of critical points, and the relation with stability, which we now define.

Definition 1.3. Let $M$ be smooth, closed manifold of dimension $n$, and $g$ a critical metric for $\tilde{\mathcal{F}}_{\tau}$. Then $g$ is called a local minimizer (for $\tilde{\mathcal{F}}_{\tau}$ ) if for all metrics $\tilde{g}$ in a $C^{2, \alpha}$-neighborhood of $g$,

$$
\tilde{\mathcal{F}}_{\tau}[\tilde{g}] \geq \tilde{\mathcal{F}}_{\tau}[g] .
$$

If, in addition, equality holds if and only if $\tilde{g}=e^{c} \phi^{*} g$ for some $C^{3, \alpha}$-diffeomorphism $\phi: M \rightarrow M$ and a constant $c \in \mathbb{R}$, then $g$ is called a strict local minimizer.

A metric $g$ is called strictly stable if the Jacobi operator is positive (as a bilinear form on $L^{2}$ ) when restricted to variations $h$ with $\delta_{g} h=\frac{1}{n} d(\operatorname{tr} h)$. If the Jacobi operator on this subspace is positive for all $h$ orthogonal to the kernel and, in addition, all kernel elements are integrable, then $g$ is called stable.

In Section 6 we show that if $g$ is strictly stable then $g$ is a strict local minimizer. Furthermore, if $g$ is stable, then $g$ is a local minimizer, see Proposition 6.2, In this latter statement, the integrability of the kernel directions is crucial, as this implication is not necessarily true otherwise, see Theorem 1.8 below.

Again, the full statement of our main theorem regarding stability for $\tilde{\mathcal{F}}_{\tau}$ is rather complicated, so for the purpose of simplicity in the introduction, we only state here the special case of $\tau>-1 / n$ :

Theorem 1.4. Let $(M, g)$ be an $n$-dimensional Einstein manifold, $n \geq 3$, and let $\tau>-1 / n$. If $R>0$ and

$$
\operatorname{spec}_{T T}\left(-\Delta_{L}\right) \cap\left[\frac{2}{n} R,\left(\frac{4}{n}+2 \tau\right) R\right]=\varnothing,
$$

then $g$ is a strict local minimizer for $\tilde{\mathcal{F}}_{\tau}$. If $R<0$ and $n=3$ or $n=4$, then the same result holds provided that endpoints in (1.8) are reversed.

Strict stability of $g$ should imply that the gradient flow for the functional will converge to $g$ for all initial metrics sufficiently close to $g$. However, we will not pursue this here, and refer the reader to [Bou10b, Str08, Str12, YYu3, Ye93] for other results regarding gradient flows for Riemannian functionals.

1.4. The Bach tensor in dimension four. We remark that for $\tau=-n / 4(n-1)$, this functional is equivalent to $\int \sigma_{2}\left(A_{g}\right) d V$, where $\sigma_{2}(A)$ denotes the second elementary symmetric function of the Schouten tensor. The conformal properties of this 
functional have been widely studied, see [Via06] for references. Critical points of this functional in dimension three were studied by the authors in in GV01.

In dimension four $(\tau=-1 / 3)$ this functional is conformally invariant, and a critical metric satisfies the Euler-Lagrange equations

$$
B_{i j} \equiv-4\left(\nabla^{k} \nabla^{l} W_{i k j l}+\frac{1}{2} R^{k l} W_{i k j l}\right)=0 .
$$

The tensor $B_{i j}$ is known as the Bach tensor [Bac21, Der83]. Any Einstein metric is necessarily Bach-flat. The Bach tensor is conformally invariant, traceless, and divergence-free.

The linearized Bach tensor fails to be elliptic due to both conformal and diffeomorphism invariance, so the condition (1.9) does not define an elliptic equation in $g$. In Section 2.3 we define a map $P_{g}^{B}: S_{0}^{2}\left(T^{*} M\right) \rightarrow S_{0}^{2}\left(T^{*} M\right)$, where $S_{0}^{2}\left(T^{*} M\right)$ consists of pointwise traceless tensors. In Section 2.3, we will show that the zeroes of $P_{g}^{B}$ correspond exactly to Bach-flat metrics in a neighborhood of $g$, up to diffeomorphism and conformal change of metric. Furthermore, $P_{g}^{B}$ has the advantange of being elliptic, so there is again a Kuranishi map $\Psi_{B}: H_{-1 / 3}^{1} \rightarrow H_{-1 / 3}^{2}$, and the moduli space is locally described by the orbit space of the isometry group acting on the zero locus $\Psi_{B}^{-1}(0)$.

Due to this extra conformal invariance, the above conditions of rigidity and strict local minimization need to be modified slightly for the Bach tensor:

Definition 1.5. Let $\left(M^{4}, g\right)$ be a Bach-flat manifold. The metric $g$ is called infinitesimally Bach-rigid if $H_{-1 / 3}^{1}=\{0\}$, and $g$ is called Bach-rigid if there exists a $C^{4, \alpha}$ neighborhood $U$ of $g$ in the space of Riemannian metrics such that if $\tilde{g} \in U$ and $\tilde{g}$ satisfies (1.9), then $\tilde{g}=e^{f} \phi^{*} g$ for some $C^{5, \alpha}$-diffeomorphism $\phi: M \rightarrow M$ and a $C^{4, \alpha}$ function $f: M \rightarrow \mathbb{R}$.

The Bach-flat metric $g$ is called a local minimizer for $\mathcal{W}$ if for all metrics $\tilde{g}$ in a $C^{2, \alpha}$-neighborhood of $g$,

$$
\mathcal{W}[\tilde{g}] \geq \mathcal{W}[g]
$$

If, in addition, equality holds in (1.10) if and only if $\tilde{g}=e^{f} \phi^{*} g$ for some $C^{3, \alpha}$ diffeomorphism $\phi: M \rightarrow M$ and $C^{2, \alpha}$-function $f: M \rightarrow \mathbb{R}$, then $g$ is a strict local minimizer for $\mathcal{W}$.

A metric $g$ is called strictly Bach-stable if the Jacobi operator is positive (as a bilinear form on $L^{2}$ ) when restricted to transverse-traceless variations $h$. If the Jacobi operator on TT-variations is positive for all $h$ orthogonal to the kernel and, in addition, all kernel elements are integrable, then $g$ is called Bach-stable.

In Section 6 we show that if $g$ is strictly Bach-stable then $g$ is a strict local minimizer of $\mathcal{W}$. Furthermore, if $g$ is Bach-stable, then $g$ is a local minimizer, see Proposition 6.4. Our main result regarding the rigidity and stability of Bach-flat metrics is the following.

Theorem 1.6. Let $\left(M^{4}, g\right)$ be an Einstein manifold. Assume that

$$
\left\{\frac{1}{3} R, \frac{1}{2} R\right\} \notin \operatorname{spec}_{T T}\left(-\Delta_{L}\right) \text {. }
$$


Then $H_{-1 / 3}^{1}=\{0\}$ and consequently $g$ is Bach-rigid.

If $R>0$, and $g$ moreover satisfies

$$
\operatorname{spec}_{T T}\left(-\Delta_{L}\right) \cap\left[\frac{1}{3} R, \frac{1}{2} R\right]=\varnothing,
$$

then $g$ is strict local minimizer for $\mathcal{W}$. The same result holds if $R<0$, provided that the endpoints of the interval in (1.12) are reversed.

We remark that the second variation of $\mathcal{W}$ was previously computed at an Einstein metric in dimension four by Kobayashi [Kob85]. The infinitesimal rigidity and stability statements follow from this computation. Our main contribution in Theorem 1.6 is therefore to conclude actual rigidity and strict local minimization from the infinitesimal statements.

1.5. Examples. We remark that the functional $\mathcal{R}=\int|R m|^{2} d V$ has been studied by many authors, see for example [And01, And97, Ber03, LeB04, Mut74, Str08. Decomposing the curvature tensor, we may write

$$
\mathcal{F}_{-1 / 2(n-1)}=\frac{n-2}{4}\{\mathcal{R}-\mathcal{W}\} .
$$

Consequently, if one can show that a metric $g$ is a local minimizer for $\tau=-1 / 2(n-1)$, then if $g$ is also known to minimize $\tilde{\mathcal{W}}$ it follows from (1.13) that $\tilde{\mathcal{R}}$ is also locally minimized. Thus the above results will also have applications to the variational structure of $\tilde{\mathcal{R}}$ in many examples.

In Section 7, we will present examples for which rigidity and stability hold. The following is a brief summary.

- The round sphere $\left(S^{n}, g_{S}\right)$ :

Theorem 1.7. On $\left(S^{n}, g_{S}\right)$, or any constant curvature quotient thereof, if $n \geq 4, g_{S}$ is a strict local minimizer for $\tilde{\mathcal{F}}_{\tau}$ provided that

$$
\frac{4-3 n}{2 n(n-1)}<\tau<\frac{2}{n(n-1)} \text {. }
$$

If $n=3$, the same conclusion holds provided that

$$
-\frac{3}{8}<\tau<\frac{1}{3}
$$

For any $n \geq 3, g_{S}$ is a strict local minimizer for $\tilde{\mathcal{R}}$.

We remark that the infinitesimal stability for $\tilde{\mathcal{R}}$ was previously shown in [Mut74.

In the case of $n=3$, there is an interesting variation of the round metric given by scaling the fibers of the Hopf fibration $S^{1} \rightarrow S^{3} \rightarrow S^{2}=\mathbb{C P}^{1}$, known as Berger spheres. In Section 7, we will employ this variation to show that the upper endpoint in Theorem 1.7 is sharp:

Theorem 1.8. For $n=3, g_{S}$ is not a local minimizer for $\tau=1 / 3$, that is, there is a path $g_{s}$ with $g_{1}=g_{S}$, with $\tilde{\mathcal{F}}_{1 / 3}\left[g_{s}\right]<\tilde{\mathcal{F}}_{1 / 3}\left[g_{S}\right]$ for $s<1$. Furthermore, there are non-trivial transverse-traceless elements $h \in H_{1 / 3}^{1}$ which are not integrable. 
As mentioned above, a similar non-integrability phenomenon occurs for infinitesimal Einstein deformations on certain Einstein manifolds [Koi82]. Our proof is similar to Koiso's in spirit: we show that the third derivative of the functional (in our case for the Berger sphere variation) is non-zero.

- Compact hyperbolic manifolds:

Theorem 1.9. Let $\left(M^{n}, g\right)$ be a compact hyperbolic manifold. If $n=3$ or $n=4$, then $g$ is a strict local minimizer for $\tilde{\mathcal{F}}_{\tau}$ provided that

$$
-\frac{1}{3}<\tau
$$

Consequently, $g$ is a strict local minimizer for $\tilde{\mathcal{R}}$. If $n \geq 5$, then $g$ is a strict local minimizer for $\tilde{\mathcal{F}}_{\tau}$ provided that

$$
\frac{4-3 n}{2 n(n-1)}<\tau \leq-\frac{1}{n}
$$

- Complex projective space $\left(\mathbb{C P}^{m}, g_{F S}\right), m \geq 2$ :

Theorem 1.10. On $\left(\mathbb{C P}^{m}, g_{F S}\right), m \geq 2$, the Fubini-Study metric $g_{F S}$ is a strict local minimizer for $\tilde{\mathcal{F}}_{\tau}$ provided that

$$
\frac{2-3 m}{2 m(2 m-1)}<\tau<\frac{1}{m(m+1)}
$$

For $n=2 m=4$, and $\tau=-1 / 3, g_{F S}$ is the unique global minimizer of $\mathcal{W}$ among the class of metrics with positive scalar curvature, up to diffeomorphism and conformal changes of metric. Furthermore, in this case, $g_{F S}$ is a strict local minimizer for $\mathcal{R}$.

The global minimization statement for $\mathcal{W}$ in dimension four follows from the signature theorem together with a result of Hitchin [Bes87].

- The product of round spheres $\left(S^{m} \times S^{m}, g_{1}+g_{2}\right)$ with $m \geq 2$ :

Theorem 1.11. On $\left(S^{m} \times S^{m}, g_{1}+g_{2}\right)$ with $m>1, g_{1}$ and $g_{2}$ round metrics with $\operatorname{Vol}\left(g_{1}\right)=\operatorname{Vol}\left(g_{2}\right)$, the product metric $g_{1}+g_{2}$ is a strict local minimizer for $\tilde{\mathcal{F}}_{\tau}$ provided that

$$
\frac{2-3 m}{2 m(2 m-1)}<\tau<\frac{2-m}{2 m(m-1)}
$$

For $n=2 m=4$ and $\tau=-1 / 3, g_{1}+g_{2}$ is the unique global minimizer of $\mathcal{W}$ among the class of positive scalar curvature metrics, up to diffeomorphism and conformal changes of metric. Furthermore, in this case, $g_{1}+g_{2}$ is a strict local minimizer for $\mathcal{R}$.

The global minimization statement for $\mathcal{W}$ was proved in Gur98]. We emphasize that $\tau=0$ is not in the included range for $m=2$. This is because there are non-trivial infinitesimal deformations for $\tau=0$ at the product metric, in fact, $\operatorname{dim}\left(H_{0}^{1}\right)=9$, 
see Section 7.4. We do not know if the right endpoint in (1.19) is optimal for strict minimization.

- The Ricci-flat case:

Ricci-flat metrics which admit nonzero parallel spinors are locally maximizing in the transverse-traceless direction as critical points of the Einstein-Hilbert functional [DWW05]. For quadratic functionals, we have the following.

Theorem 1.12. Let $(M, g)$ be a compact Ricci-flat manifold, and assume that all infinitesimal Einstein deformations are integrable. Then $g$ is a local minimizer for $\tilde{\mathcal{F}}_{\tau}$ provided that

$$
-\frac{n}{4(n-1)}<\tau
$$

In particular, this holds if $(M, g)$ is a flat torus, or if $(M, g)$ is a Calabi-Yau metric.

Obviously, such a metric is minimizing if $\tau \geq-1 / n$, so the main point is that this can be improved, at least locally. The integrability of infinitesimal Einstein deformations on a Calabi-Yau manifold was proved by Bogomolov and Tian Bog78, Tia87.

1.6. Reverse Bishop's inequalities. The classical Bishop's inequality implies that if $(M, g)$ is a closed manifold with $\operatorname{Ric}(g) \geq \operatorname{Ric}\left(S^{n}\right)=(n-1) g$, then the volume satisfies $\operatorname{Vol}(g) \leq \operatorname{Vol}\left(S^{n}\right)$, and equality holds only if $(M, g)$ is isometric to the sphere. An interesting consequence of stability for $\tau=0$ for Einstein metrics is that, locally, a "reverse Bishop's inequality" holds:

Theorem 1.13. Let $(M, g)$ be an Einstein manifold with positive scalar curvature, normalized so that $\operatorname{Ric}(g)=(n-1) \mathrm{g}$. Assume $g$ is a strict local minimizer for $\tilde{\mathcal{F}}_{0}$. Then there exists a $C^{2, \alpha}$-neighborhood $U$ of $g$ such that if $\tilde{g} \in U$ with $\operatorname{Ric}(\tilde{g}) \leq(n-1) \tilde{g}$, then $\operatorname{Vol}(\tilde{g}) \geq \operatorname{Vol}(g)$ with equality if and only if $\tilde{g}=\phi^{*} g$ for some diffeomorphism $\phi: M \rightarrow M$.

From the list of examples above, we see that if $(M, g)$ is a sphere, space form, or complex projective space, then for metrics $\tilde{g}$ near $g$

$$
\operatorname{Ric}(\tilde{g}) \leq \operatorname{Ric}(g) \Longrightarrow \operatorname{Vol}(M, \tilde{g}) \geq \operatorname{Vol}(M, g) .
$$

Based on the above results for spheres and complex projective spaces, we conjecture that if $\left(M^{n}, g\right)$ is a compact rank one symmetric space, then $g$ is a strict local minimizer for $\tilde{\mathcal{F}}_{0}$. This would imply that Theorem 1.13 also holds for quaternionic projective spaces and the Cayley plane.

Theorem 1.13 has a counterpart for negative Einstein manifolds, see Corollary 6.5. We mention that on certain negative Einstein manifolds, such as hyperbolic or KählerEinstein manifolds, there are important global results which in some cases are much stronger than what our local analysis can yield. One example is the work of BessonCourtois-Gallot ([BCG95], [BCG99]) on volume rigidity: If $\left(M^{n}, g_{0}\right)$ is a compact 
hyperbolic manifold, and $g$ is any Riemannian metric on $M^{n}$ then

$$
\operatorname{Ric}(g) \geq-(n-1) g \Longrightarrow \operatorname{Vol}(g) \geq \operatorname{Vol}\left(g_{0}\right)
$$

with equality if and only if $g$ is isometric to $g_{0}$. Note the requirement that $M^{n}$ support a hyperbolic metric: in particular, this gives a global result for any negative Einstein three-manifold, but does not give (even local) information in dimensions $n \geq 4$ when $M^{n}$ is only known to admit an Einstein metric. We also mention the article [DWW07]. In this work, a local volume comparison theorem is proved using only the scalar curvature, at a negative Kähler-Einstein metric.

1.7. Global minimization. The functionals $\int|R i c|^{2}$ and $\int R^{2}$ are known to be globally minimized in dimension four at a negative Kähler-Einstein metric. This is proved in [LeB01] using Seiberg-Witten theory, along with many other interesting results regarding minimal volumes.

In any odd dimension, the fibration $S^{1} \rightarrow S^{2 n+1} \rightarrow \mathbb{C P}^{n}$ has totally geodesic fibers. Thus by scaling the fiber, there are metrics which collapse with bounded curvature on odd-dimensional spheres [Ber03, Chapter 11], where it is also pointed out that the global minimum of $\tilde{\mathcal{R}}$ is equal to zero in dimensions $n \geq 5$ on any Riemannian manifold. Thus the spherical metric is most definintely not a global minimizer of $\tilde{\mathcal{F}}_{0}$ in dimensions other than four. However, we conjecture that the round sphere $\left(S^{4}, g_{S}\right)$ is a global minimizer of $\mathcal{F}_{0}$.

1.8. Other functionals. Many of the results in this paper hold for arbitrary Riemannian functionals, e.g., Proposition 6.2. For example, one can also consider other $L^{p}$-norms of the curvature. The scale-invariant $L^{n / 2}$-norm of the curvature carries topological information, due to the Chern-Gauss-Bonnet Theorem. We point out that many of the above local minimization theorems imply local minimization for the scale-invariant norm simply by Hölder's inequality when $n \geq 4$. For example, for the functional $\tilde{\mathcal{F}}_{0}$,

$$
\tilde{\mathcal{F}}_{0}[g]=(V o l)^{4 / n-1} \int|R i c|^{2} d V \leq\left\{\int|R i c|^{n / 2} d V\right\}^{4 / n} .
$$

Thus a local minimizer for $\tilde{\mathcal{F}}_{0}$ is automatically a local minimizer for the scale-invariant norm of the Ricci tensor. Thus many of the above results can be restated for the scaleinvariant power (but we do not explicitly restate each case separately here).

1.9. Acknowledgements. The authors would like to thank Gang Tian for helpful remarks regarding the various "slicing" lemmas in the paper. Thanks are also due to Robert Bryant, Claude LeBrun, and Peter Li for helpful comments.

Finally, the authors would also thank the referee, whose careful reading of the manuscript resulted in a number of improvements to the exposition. 


\section{LocAl PROPERTiES OF THE MOduli SPACE}

In this Section we recall some basic variational formulae, all of which are found in [Bes87, Chapter 4]. We begin with the squared $L^{2}$-norm of Ricci,

$$
\mathcal{F}_{0}[g]=\int|R i c|^{2} d V=\int g^{i p} g^{j q} R_{p q} R_{i j} d V .
$$

Along an arbitrary path $g_{s}$, let $g_{s}^{\prime}=\frac{d}{d s} g_{s}$. We have

$$
\frac{d}{d s} \mathcal{F}_{0}[g]=\int g^{i p} g^{j q}\left(g_{s}^{\prime}\right)_{i j}\left(\nabla \mathcal{F}_{0}\right)_{p q} d V
$$

where

$$
\left(\nabla \mathcal{F}_{0}\right)_{p q}=-\Delta_{L}(R i c)_{p q}+\nabla_{p} \nabla_{q} R-\frac{1}{2}(\Delta R) g_{p q}-2 R_{p}^{l} R_{l q}+\frac{1}{2}|R i c|^{2} g_{p q}
$$

Recalling that

$$
\left(\Delta_{L} h\right)_{i j}=(\Delta h)_{i j}+2 R_{i p j q} h^{p q}-R_{i}^{l} h_{l j}-R_{j}^{l} h_{i l}
$$

the gradient is also written as

$$
\left(\nabla \mathcal{F}_{0}\right)_{p q}=-\Delta(R i c)_{p q}-2 R_{p k q l} R^{k l}+\nabla_{p} \nabla_{q} R-\frac{1}{2}(\Delta R) g_{p q}+\frac{1}{2}|R i c|^{2} g_{p q} .
$$

Proposition 2.1. If $g$ is Einstein with Ric $=(R / n) g$, then

$$
\nabla \mathcal{F}_{0}[g]=\frac{n-4}{2 n^{2}} R^{2} g
$$

Proof. A simple computation from the above formula.

Next, we consider the functional

$$
\mathcal{S}[g]=\int R^{2} d V
$$

We have

$$
\frac{d}{d s} \mathcal{S}[g]=\int g^{i p} g^{j q}\left(g_{s}^{\prime}\right)_{i j}(\nabla \mathcal{S})_{p q} d V
$$

where

$$
(\nabla \mathcal{S})_{p q}=2 \nabla_{p} \nabla_{q} R-2(\Delta R) g_{p q}-2 R R_{p q}+\frac{1}{2} R^{2} g_{p q}
$$

Proposition 2.2. If $g$ is Einstein with Ric $=(R / n) g$, then

$$
\nabla \mathcal{S}[g]=\frac{n-4}{2 n} R^{2} g .
$$

Proof. Another simple computation. 
2.1. Volume normalized functionals. The functional $\mathcal{F}_{\tau}$ is not scale-invariant in dimensions other than four. A standard remedy for this is to normalize the functionals by a suitable power of the volume, which is equivalent to restricting the functionals to the space of unit volume metrics $\mathcal{M}_{1}$. Therefore, we need to understand the effect of the normalizing term on the formulas for the first and second variation of $\mathcal{F}_{\tau}$. The following is elementary:

Claim 2.3. The functional

$$
\tilde{\mathcal{F}}_{\tau}[g]=(\operatorname{Vol}(g))^{\frac{4}{n}-1} \cdot \mathcal{F}_{\tau}[g]
$$

is scale-invariant.

In the following, we let $p=\frac{4}{n}-1$. Let $g_{s}$ denote a path of metrics through $g=g_{0}$.

Lemma 2.4. The first and second variation of the volume are

$$
\begin{aligned}
& \left(\operatorname{Vol}\left(g_{s}\right)\right)^{\prime}=\frac{1}{2} \int_{M}\left(\operatorname{tr}_{g_{s}} g_{s}^{\prime}\right) d V \\
& \left(\operatorname{Vol}\left(g_{s}\right)\right)^{\prime \prime}=-\frac{1}{2} \int_{M}\left|g_{s}^{\prime}\right|^{2} d V+\frac{1}{2} \int_{M}\left(\operatorname{tr}_{g_{s}} g_{s}^{\prime \prime}\right) d V+\frac{1}{4} \int\left(\operatorname{tr}_{g_{s}} g_{s}^{\prime}\right)^{2} d V .
\end{aligned}
$$

Proof. See [Bes87], Chapter 4.

Next, we consider the first variation of $\tilde{\mathcal{F}}_{\tau}$ :

Lemma 2.5. A critical metric of $\tilde{\mathcal{F}}_{\tau}$ satisfies

$$
\begin{aligned}
\mathcal{F}_{\tau}^{\prime} & =-p(V o l)^{-1}(V o l)^{\prime} \cdot \mathcal{F}_{\tau}, \\
\nabla \mathcal{F}_{\tau} & =-\frac{p}{2} V o l^{-1} \cdot \mathcal{F}_{\tau} \cdot g_{s},
\end{aligned}
$$

where $\nabla \mathcal{F}_{\tau}$ denotes the gradient of $\mathcal{F}_{\tau}$,

Proof. Differentiating the functional, we compute

$$
\left(\tilde{\mathcal{F}}_{\tau}\left[g_{s}\right]\right)^{\prime}=p(V o l)^{p-1}(V o l)^{\prime} \mathcal{F}_{\tau}+(V o l)^{p}\left(\mathcal{F}_{\tau}\left[g_{s}\right]\right)^{\prime}
$$

Therefore a critical point of $\tilde{\mathcal{F}}_{\tau}$ clearly satisfies (2.14). We also can write this as

$$
\begin{aligned}
\left(\tilde{\mathcal{F}}_{\tau}\left[g_{s}\right]\right)^{\prime} & =p(V o l)^{p-1} \mathcal{F}_{\tau} \cdot \frac{1}{2} \int_{M}\left(t r_{g_{s}} g_{s}^{\prime}\right) d V+(V o l)^{p} \int_{M}\left\langle\nabla \mathcal{F}_{\tau}, g_{s}^{\prime}\right\rangle d V \\
& =V_{o l} l^{p}\left(\int\left\langle\nabla \mathcal{F}_{\tau}+\frac{p}{2} V o l^{-1} \cdot \mathcal{F}_{\tau} \cdot g_{s}, h\right\rangle d V\right)
\end{aligned}
$$

Consequently, a critical point of $\tilde{\mathcal{F}}_{\tau}$ also satisfies (2.15). 
2.2. Local structure of the moduli space. To understand to local properties of the moduli space of critical metrics, we need to cast the equation in the framework of Fredholm theory. This requires an elliptic operator, so one must "gauge" the equation to become elliptic.

We begin with some definitions. For simplicity of notation, we will treat the domain and range of an operator as if it were the bundle itself, although the operator really acts on sections of the bundle. Let $\delta_{g}: S^{2}\left(T^{*} M\right) \rightarrow T^{*} M$ denote the divergence operator

$$
\left(\delta_{g} h\right)_{j}=\nabla^{i} h_{i j}
$$

and $\delta^{*}: T^{*} M \rightarrow S^{2}\left(T^{*} M\right)$ its $L^{2}$-adjoint. Note that

$$
\delta^{*}=-\frac{1}{2} \mathcal{L}
$$

where $\mathcal{L}$ is the Killing operator:

$$
\left(\mathcal{L}_{g} \omega\right)_{i j}=\nabla_{i} \omega_{j}+\nabla_{j} \omega_{i}
$$

We let $\mathcal{K}_{g}$ denote the conformal Killing operator, the trace-free part of $\mathcal{L}_{g}$ :

$$
\left(\mathcal{K}_{g} \omega\right)_{i j}=\nabla_{i} \omega_{j}+\nabla_{j} \omega_{i}-\frac{2}{n}\left(\delta_{g} \omega\right) g_{i j}
$$

Note that if

$$
\beta_{g} h=\delta_{g} h-\frac{1}{n} d(\operatorname{tr} h),
$$

then

$$
\beta^{*}=-\frac{1}{2} \mathcal{K}
$$

Let $g$ be a critical metric for the normalized functional

$$
\tilde{\mathcal{F}}_{\tau}=(V o l)^{\frac{4}{n}-1} \int\left(|R i c|^{2}+\tau R^{2}\right) d V .
$$

Assume $\mathcal{U} \subset S^{2}\left(T^{*} M\right)$ is a neighborhood of the zero section, sufficiently small so that $\theta \in \mathcal{U}_{0} \Rightarrow \tilde{g}=g+\theta$ is a metric. Consider the map $P_{g}: \mathcal{U} \rightarrow S^{2}\left(T^{*} M\right)$ given by

$$
P_{g}(\theta)=\nabla \tilde{\mathcal{F}}_{\tau}(g+\theta)+\frac{1}{2} \mathcal{K}_{g+\theta}\left[\beta_{g} \mathcal{K}_{g} \beta_{g} \theta\right]-\gamma(\theta) \cdot g,
$$

where

$$
\gamma(\theta)=\frac{1}{n} \operatorname{Vol}(g)^{-1} \int \operatorname{tr}_{g}\left\{\nabla \tilde{\mathcal{F}}_{\tau}(g+\theta)\right\} d V_{g}
$$

The term involving $\gamma$ is included to make the trace of $P$ have mean value zero (with respect to $g$ ); hence

$$
P_{g}: \mathcal{U}_{0} \rightarrow \bar{S}_{0}^{2}\left(T^{*} M\right)
$$

where

$$
\mathcal{U}_{0}=\mathcal{U} \cap \bar{S}_{0}^{2}\left(T^{*} M\right)
$$


and $\bar{S}_{0}^{2}\left(T^{*} M\right)$ is defined in (1.5) . The domain of $P_{g}$ will be tensors in $\mathcal{U}_{0}$ of Hölder class $C^{k+1, \alpha}$. The term involving the operator $\mathcal{K}$ is included to handle gauge-invariance. To simplify notation, we denote

$$
\square_{\mathcal{K}_{g}} \omega=\beta_{g} \mathcal{K}_{g} \omega=-\frac{1}{2} \mathcal{K}_{g}^{*} \mathcal{K}_{g} \omega
$$

so that the gauge-fixing term can be written

$$
\frac{1}{2} \mathcal{K}_{g+\theta}\left[\beta_{g} \mathcal{K}_{g} \beta_{g} \theta\right]=\frac{1}{2} \mathcal{K}_{g+\theta}\left[\square_{\mathcal{K}_{g}} \beta_{g} \theta\right] .
$$

Note that the kernel of $\square_{\mathcal{K}_{g}}$ is precisely the space of conformal Killing forms (with respect to $g$ ), since

$$
\left\langle\omega, \square_{\mathcal{K}_{g}} \omega\right\rangle_{L^{2}}=-\frac{1}{2}\left\|\mathcal{K}_{g} \omega\right\|_{L^{2}}^{2} .
$$

Lemma 2.6. The operator $\square_{\mathcal{K}_{g}}: T^{*} M \rightarrow T^{*} M$ is elliptic for $n \neq 2$.

Proof. Since $\mathcal{K}_{g} \omega$ is traceless, we have

$$
\left(\square_{\mathcal{K}_{g}} \omega\right)_{j}=(\Delta \omega)_{j}+\nabla^{i} \nabla_{j} \omega_{i}-\frac{2}{n} \nabla_{j} \nabla^{i} \omega_{i} .
$$

Consequently, the symbol is given by

$$
(\xi, \omega) \mapsto|\xi|^{2} \omega_{j}+\left(1-\frac{2}{n}\right) \xi_{j}\left(\xi_{i} \omega_{i}\right)
$$

Pairing this with $\xi_{j}$ shows injectivity for $\xi \neq 0$.

The main result of this section is that the moduli space of critical metrics is precisely given by the zero-locus of $P_{g}$ :

Theorem 2.7. Assume

$$
\tau \neq-\frac{n}{4(n-1)}
$$

and consider $P_{g}$ as a mapping from $C^{k+1, \alpha}$ to $C^{k-3, \alpha}$ for $k \geq 3$. Then:

(i) The linearization of $P_{g}$ at $\theta=0$ is an elliptic operator.

(ii) If $\theta$ is sufficiently small and $P_{g}(\theta)=0$, then the metric $\tilde{g}=g+\theta$ is a smooth Riemannian metric and is critical for the normalized functional $\tilde{\mathcal{F}}_{\tau}$.

(iii) Conversely, if $g_{1}=g+\theta_{1}$ is a critical metric in a sufficiently small $C^{k+1, \alpha}{ }_{-}$ neighborhood of $g(k \geq 3)$, then there exists a $C^{k+2, \alpha}$-diffeomorphism $\phi: M \rightarrow M$ and a constant $c$ such that

$$
e^{c} \phi^{*} g_{1}=g+\tilde{\theta}
$$

with

$$
P_{g}(\tilde{\theta})=0
$$

and

$$
\int \operatorname{tr}_{g} \tilde{\theta} d V_{g}=0 .
$$


Remark 2.8. When $\tau=\tau_{c}=-\frac{n}{4(n-1)}$, then

$$
\tilde{\mathcal{F}}_{\tau_{c}}[g]=-2(n-2)^{2} \operatorname{Vol}(g)^{\frac{4}{n}-1} \int \sigma_{2}\left(A_{g}\right) d V_{g}
$$

When $n \neq 4$, a metric $g$ is critical over all conformal variations which preserve the volume if and only if

$$
\sigma_{2}\left(A_{g}\right)=\text { const., }
$$

a second order condition in the metric (for an explanation of the notation, see Section 1.4 in the Introduction). When $n=4$, the integral in (2.35) is conformally invariant (hence the gradient is trace-free). In both cases, this reduction in the order of the Euler equation results in a degeneracy on the symbol level.

Remark 2.9. The choice of the "gauge-fixing" term is not unique. For the linearized Einstein equations, one can choose a gauge-fixing term which reduces the symbol to that of the rough Laplacian (see [GL91]). Such a simplification does not seem possible for the linearized gradient of (2.23).

Proof of Theorem 2.7. We will postpone the proof of ellipticity, as it involves a rather lengthy calculation of the symbol. To prove part $(i i)$, suppose

$$
0=P_{g}(\theta)=\nabla \tilde{\mathcal{F}}_{\tau}(g+\theta)+\frac{1}{2} \mathcal{K}_{g+\theta}\left[\square_{\mathcal{K}_{g}} \beta_{g} \theta\right]-\gamma(\theta) \cdot g
$$

Let $\tilde{g}=g+\theta$. If we trace (2.36) with respect to $\tilde{g}$ and integrate,

$$
\begin{aligned}
0 & =\int \operatorname{tr}_{\tilde{g}}\left[\nabla \tilde{\mathcal{F}}_{\tau}(\tilde{g})\right] d V_{\tilde{g}}+\frac{1}{2} \int \operatorname{tr}_{\tilde{g}}\left[\mathcal{K}_{\tilde{g}}\left[\square_{\mathcal{K}_{g}} \beta_{g} \theta\right]\right] d V_{\tilde{g}}-\gamma(\theta) \int \operatorname{tr}_{\tilde{g}} g d V_{\tilde{g}} \\
& =\int \operatorname{tr}_{\tilde{g}}\left[\nabla \tilde{\mathcal{F}}_{\tau}(\tilde{g})\right] d V_{\tilde{g}}-\gamma(\theta) \int \operatorname{tr}_{\tilde{g}} g d V_{\tilde{g}} .
\end{aligned}
$$

Here we used the fact that $\mathcal{K}[\cdot]$ is trace-free. Also, since $\tilde{\mathcal{F}}_{\tau}$ is scale-invariant,

$$
\tilde{\mathcal{F}}_{\tau}[\tilde{g}]=\tilde{\mathcal{F}}_{\tau}\left[e^{r} \tilde{g}\right], r \in \mathbb{R} .
$$

Differentiating this identity at $r=0$,

$$
\begin{aligned}
0 & =\left.\frac{d}{d r} \tilde{\mathcal{F}}_{\tau}\left[e^{r} \tilde{g}\right]\right|_{r=0}=\int\left\langle\nabla \tilde{\mathcal{F}}_{\tau}(\tilde{g}),\left.\frac{d}{d r}\left(e^{r} \tilde{g}\right)\right|_{r=0}\right\rangle d V_{\tilde{g}} \\
& =\int\left\langle\nabla \tilde{\mathcal{F}}_{\tau}(\tilde{g}), \tilde{g}\right\rangle d V_{\tilde{g}}=\int \operatorname{tr}_{\tilde{g}}\left[\nabla \tilde{\mathcal{F}}_{\tau}(\tilde{g})\right] d V_{\tilde{g}} .
\end{aligned}
$$

Therefore, by (2.37) we conclude

$$
\gamma(\theta) \int \operatorname{tr}_{\tilde{g}} g d V_{\tilde{g}}=0
$$

Since $g$ is positive-definite,

$$
\int \operatorname{tr}_{\tilde{g}} g d V_{\tilde{g}}>0
$$


we conclude that $\gamma(\theta)=0$. Therefore, by the definition of $P$ in (2.24),

$$
0=\nabla \tilde{\mathcal{F}}_{\tau}(g+\theta)+\frac{1}{2} \mathcal{K}_{g+\theta}\left[\square_{\mathcal{K}_{g}} \beta_{g} \theta\right] .
$$

The next step involves an integration by parts argument, but this presents a difficulty since $\theta \in C^{4, \alpha}$ only implies that $P_{g}(\theta)$ is $C^{\alpha}$, and not necessarily differentiable. To get around this problem we mollify $\theta$; i.e., let $\left\{\theta_{\epsilon}\right\}$ be a family of smooth tensor fields such that $\theta_{\epsilon} \rightarrow \theta$ in $C^{4, \alpha}$ as $\epsilon \rightarrow 0$, and let $\tilde{g}_{\epsilon}=g+\theta_{\epsilon}$. From (2.39) and the continuity of $P$ it follows that

$$
\eta_{\epsilon}=\nabla \tilde{\mathcal{F}}_{\tau}\left(g+\theta_{\epsilon}\right)+\frac{1}{2} \mathcal{K}_{g+\theta_{\epsilon}}\left[\square_{\mathcal{K}_{g}} \beta_{g} \theta_{\epsilon}\right]
$$

where $\eta_{\epsilon} \rightarrow 0$ in $C^{\alpha}$. Pair both sides of $(2.40)$ with $\mathcal{L}_{\tilde{g}_{\epsilon}}\left[\square_{\mathcal{K}_{g}} \beta_{g} \theta_{\epsilon}\right]$ (with respect to the $L^{2}$-inner product defined by $\left.\tilde{g}_{\epsilon}\right)$, where $\mathcal{L}$ is the Killing operator defined in (2.20):

$$
\begin{aligned}
\left\langle\mathcal{L}_{\tilde{g}_{\epsilon}}\left[\square_{\mathcal{K}_{g}} \beta_{g} \theta_{\epsilon}\right], \eta_{\epsilon}\right\rangle_{L^{2}} & =\left\langle\mathcal{L}_{\tilde{g}_{\epsilon}}\left[\square_{\mathcal{K}_{g}} \beta_{g} \theta_{\epsilon}\right], \nabla \tilde{\mathcal{F}}_{\tau}\left(\tilde{g}_{\epsilon}\right)+\frac{1}{2} \mathcal{K}_{\tilde{g}_{\epsilon}}\left[\square_{\mathcal{K}_{g}} \beta_{g} \theta_{\epsilon}\right]\right\rangle_{L^{2}} \\
& =\left\langle\mathcal{L}_{\tilde{g}_{\epsilon}}\left[\square_{\mathcal{K}_{g}} \beta_{g} \theta_{\epsilon}\right], \nabla \tilde{\mathcal{F}}_{\tau}\left(\tilde{g}_{\epsilon}\right)\right\rangle_{L^{2}}+\frac{1}{2}\left\|\mathcal{K}_{\tilde{g}_{\epsilon}}\left[\square_{\mathcal{K}_{g}} \beta_{g} \theta_{\epsilon}\right]\right\|_{L^{2}}^{2} .
\end{aligned}
$$

Integrating by parts in the first term on the right-hand side, we obtain

$$
\left\langle\mathcal{L}_{\tilde{g}_{\epsilon}}\left[\square_{\mathcal{K}_{g}} \beta_{g} \theta_{\epsilon}\right], \nabla \tilde{\mathcal{F}}_{\tau}\left(\tilde{g}_{\epsilon}\right)\right\rangle_{L^{2}}=-2\left\langle\square_{\mathcal{K}_{g}} \beta_{g} \theta_{\epsilon}, \delta_{\tilde{g}_{\epsilon}}\left(\nabla \tilde{\mathcal{F}}_{\tau}\left(\tilde{g}_{\epsilon}\right)\right)\right\rangle_{L^{2}}=0,
$$

since $\mathcal{L}^{*}=-2 \delta$ and the gradient of a Riemannian functional is always divergence-free (see [Bes87], Proposition 4.11). Therefore,

$$
\left\langle\mathcal{L}_{\tilde{g}_{\epsilon}}\left[\square_{\mathcal{K}_{g}} \beta_{g} \theta_{\epsilon}\right], \eta_{\epsilon}\right\rangle_{L^{2}}=\frac{1}{2}\left\|\mathcal{K}_{\tilde{g}_{\epsilon}}\left[\square_{\mathcal{K}_{g}} \beta_{g} \theta_{\epsilon}\right]\right\|_{L^{2}}^{2}
$$

Letting $\epsilon \rightarrow 0$, the left-hand side converges to zero, while the right-hand side converges to $\mathcal{K}_{\tilde{g}}\left[\square_{\mathcal{K}_{g}} \beta_{g} \theta\right]$, which consequently vanishes. By (2.39) we conclude that

$$
\nabla \tilde{\mathcal{F}}_{\tau}(\tilde{g})=0
$$

as claimed.

Next, taking a trace of (2.41), yields an equation of the form

$$
\Delta_{\tilde{g}} R_{\tilde{g}}=R m_{\tilde{g}} * R m_{\tilde{g}}+\lambda \cdot \tilde{g} .
$$

Since $\tilde{g}$ is assumed to be only $C^{4, \alpha}$, around any point $p \in M$, there is a coordinate system $\left\{x^{i}\right\}$ so that the component functions $\tilde{g}_{i j}$ are $C^{4, \alpha}$. Equation (2.42) is easily seen to be an elliptic equation, and by elliptic regularity we conclude that $R_{\tilde{g}} \in C^{4, \alpha}$. The equation (2.41) then implies that $\Delta_{\tilde{g}} \operatorname{Ric}_{\tilde{g}} \in C^{2, \alpha}$, which implies that $\operatorname{Ric}_{\tilde{g}} \in C^{4, \alpha}$. Since $\tilde{g} \in C^{4, \alpha}$, there exists a harmonic coordinate system $\left\{y^{i}\right\}$ around $p$ such that the equation

$$
\frac{1}{2} \tilde{g}^{i j} \partial_{i j}^{2} \tilde{g}_{k l}+Q_{k l}(\partial \tilde{g}, \tilde{g})=-R i c_{k l}(\tilde{g})
$$

where $Q(\partial \tilde{g}, \tilde{g})$ is an expression that is quadratic in $\partial \tilde{g}$, polynomial in $\tilde{g}$ and has $\sqrt{|\tilde{g}|}$ in its denominator [Pet06]. From this we conclude that $\tilde{g}_{i j} \in C^{5, \alpha}$. A bootstrap argument shows that $\tilde{g}_{i j} \in C^{\ell, \alpha}$ for any $\ell>0$. 
Part (iii) follows from a result, known as "Ebin-Palais slicing" Ebi70, Pal]. Our treatment is based on an argument of Cheeger-Tian [CT94, Theorem 3.1], see also FM77].

Lemma 2.10. For each metric $g_{1}$ in a sufficiently small $C^{\ell+1, \alpha}$-neighborhood of $g$ $(\ell \geq 1)$, there is a $C^{\ell+2, \alpha}$-diffeomorphism $\phi: M \rightarrow M$ and a constant $c$ such that

$$
\tilde{\theta} \equiv e^{c} \phi^{*} g_{1}-g
$$

satisfies

$$
\beta_{g} \tilde{\theta}=0
$$

where $\beta_{g}$ is defined in (2.22), and

$$
\int \operatorname{tr}_{g} \tilde{\theta} d V_{g}=0
$$

Moreover, we have the estimate

$$
\|\tilde{\theta}\|_{C^{\ell+1, \alpha}} \leq C\left\|\theta_{1}\right\|_{C^{\ell+1, \alpha}}
$$

where $C=C(g)$.

Proof. Let $\left\{\omega_{1}, \ldots, \omega_{\kappa}\right\}$ denote a basis of the space of conformal Killing forms with respect to $g$. Consider the map

$$
\mathcal{N}: C^{\ell+2, \alpha}(T M) \times \mathbb{R} \times \mathbb{R}^{\kappa} \times C^{\ell+1, \alpha}\left(S^{2}\left(T^{*} M\right)\right) \rightarrow C^{\ell, \alpha}\left(T^{*} M\right) \times \mathbb{R}
$$

given by

$$
\begin{aligned}
& \mathcal{N}(X, r, v, \theta)=\mathcal{N}_{\theta}(X, r, v) \\
& =\left(\beta_{g}\left[\phi_{X, 1}^{*}(g+\theta)\right]+\sum_{i} v_{i} \omega_{i}, e^{r} \int \operatorname{tr}_{g}\left[\phi_{X, 1}^{*}(g+\theta)\right] d V_{g}-n V o l(g)\right),
\end{aligned}
$$

where $\phi_{X, 1}$ denotes the diffeomorphism obtained by following the flow generated by the vector field $X$ for unit time. It is clear that $\mathcal{N}$ is a continuously differentiable mapping, and linearizing in $(X, r, v)$ at $(X, r, v, \theta)=(0,0,0,0)$, we find

$$
\begin{aligned}
\mathcal{N}_{0}^{\prime}(Y, s, a) & =\left.\frac{d}{d \epsilon}\left(\beta_{g}\left[\phi_{\epsilon Y, 1}^{*}(g)\right]+\sum_{i}\left(\epsilon a_{i}\right) \omega_{i}, e^{\epsilon s} \int \operatorname{tr}_{g}\left[\phi_{\epsilon Y, 1}^{*}(g)\right] d V_{g}-n \operatorname{Vol}(g)\right)\right|_{\epsilon=0} \\
& =\left(\beta_{g}\left[\mathcal{L}_{g} Y^{b}\right]+\sum_{i} a_{i} \omega_{i}, n V o l(g) \cdot s\right) \\
& =\left(\square_{\mathcal{K}_{g}} Y^{b}+\sum_{i} a_{i} \omega_{i}, n V o l(g) \cdot s\right),
\end{aligned}
$$

where $Y^{b}$ is the dual one-form to $Y$.

The adjoint map $\left(\mathcal{N}_{0}^{\prime}\right)^{*}: C^{m+2, \alpha}\left(T^{*} M\right) \times \mathbb{R} \rightarrow C^{m, \alpha}(T M) \times \mathbb{R} \times \mathbb{R}^{\kappa}$ is given by

$$
\left(\mathcal{N}_{0}^{\prime}\right)^{*}(\eta, s)=\left(\left(\square_{\mathcal{K}_{g}} \eta\right)^{\sharp}, n V o l(g) s, \int\left\langle\eta, \omega_{i}\right\rangle d V\right),
$$


where $\left(\square_{\mathcal{K}_{g}} \eta\right)^{\sharp}$ is the vector field dual to $\square_{\mathcal{K}_{g}} \eta$. Setting $\left(\mathcal{N}_{0}^{\prime}\right)^{*}(\eta, s)=0$, we get $s=0$ and

$$
\begin{aligned}
\square_{\mathcal{K}_{g}} \eta & =0, \\
\int\left\langle\eta, \omega_{i}\right\rangle d V & =0,1 \leq i \leq \kappa .
\end{aligned}
$$

The first equation implies that $\eta$ is a conformal Killing field, while the second implies that $\eta$ is orthogonal (in $L^{2}$ ) to the space of conformal Killing forms. It follows that $\eta=0$, so the map $\mathcal{N}_{0}^{\prime}$ is surjective. By a standard implicit function theorem argument, given $\theta_{1} \in C^{\ell+1, \alpha}\left(S^{2}\left(T^{*} M\right)\right)$ small enough we can solve the equation $\mathcal{N}_{\theta_{1}}=(0,0)$; i.e., there is a vector field $X \in C^{\ell+2, \alpha}(T M)$, a $v \in \mathbb{R}^{\kappa}$, and a real number $r$ such that

$$
\beta_{g}\left[\phi^{*} g_{1}\right]+\sum_{i} v_{i} \omega_{i}=0, \quad e^{r} \int \operatorname{tr}_{g}\left[\phi_{X, 1}^{*}\left(g_{1}\right)\right] d V_{g}=n \operatorname{Vol}(g)
$$

where $\phi=\phi_{X, 1}$. In addition, if we let

$$
\tilde{\theta}=e^{r} \phi^{*} g_{1}-g
$$

then $\tilde{\theta}$ satisfies (2.47).

For the first equation in (2.50), fix $1 \leq \ell \leq \kappa$ and pair both sides (in $L^{2}$ ) with $\omega_{\ell}$; then $v_{\ell}=0$ since $\beta_{g}^{*} \omega_{\ell}=0$. Therefore, $\beta_{g}\left[\phi^{*} g_{1}\right]=0$. Also,

$$
\beta_{g} \tilde{\theta}=\beta_{g}\left[e^{r} \phi^{*} g_{1}-g\right]=0,
$$

which proves (2.45). The trace condition (2.46) follows from the second condition in (2.50):

$$
\begin{aligned}
\int \operatorname{tr}_{g} \tilde{\theta} d V_{g} & =\int t r_{g}\left[e^{r} \phi^{*} g_{1}-g\right] d V_{g}=\int\left\{e^{r} \cdot \operatorname{tr}_{g}\left[\phi^{*} g_{1}\right]-n\right\} d V_{g} \\
& =e^{r} \int t r_{g}\left[\phi^{*} g_{1}\right] d V_{g}-n V \operatorname{Vol}(g)=0 .
\end{aligned}
$$

To prove the theorem, suppose $g_{1}$ is a critical metric which is $C^{k+1, \alpha}$-close to $g$, for some $k \geq 3$ :

$$
\nabla \tilde{\mathcal{F}}_{\tau}\left(g_{1}\right)=0
$$

Applying the lemma, we obtain a $C^{k+2, \alpha}$-diffeomorphism $\phi: M \rightarrow M$ and a constant $c$ such that $\tilde{\theta}=e^{c} \phi^{*} g_{1}-g$ satisfies (2.34). Also, since $\tilde{\theta}$ satisfies (2.45),

$$
P_{g}(\tilde{\theta})=\nabla \tilde{\mathcal{F}}_{\tau}(g+\tilde{\theta})+\frac{1}{2} \mathcal{K}_{g+\tilde{\theta}}\left[\square_{\mathcal{K}_{g}} \beta_{g} \tilde{\theta}\right]=\nabla \tilde{\mathcal{F}}_{\tau}\left(\phi^{*} g_{1}\right)=\phi^{*}\left\{\nabla \tilde{\mathcal{F}}_{\tau}\left(g_{1}\right)\right\}=0,
$$

verifying (2.33).

To verify ellipticity, let $S_{g}: S^{2}\left(T^{*} M\right) \rightarrow S^{2}\left(T^{*} M\right)$ denote the linearization of $P_{g}$ at $\theta=0$ :

$$
S_{g} h=\left.\frac{d}{d s} P_{g}(g+s h)\right|_{s=0}=\left(\nabla \tilde{\mathcal{F}}_{\tau}\right)_{g}^{\prime}(h)+\frac{1}{2} \mathcal{K}_{g}\left[\square_{\mathcal{K}_{g}} \beta_{g} h\right] .
$$


Note that

$$
\gamma^{\prime}(0)=\left.\frac{d}{d s} \gamma(s h)\right|_{s=0}=0
$$

at a critical metric, since linearizing the definition (and using $\nabla \tilde{\mathcal{F}}_{\tau}[g]=0$ ) gives

$$
\gamma^{\prime}(0)=\frac{1}{n} \operatorname{Vol}(g)^{-1} \int \operatorname{tr}_{g}\left[\left(\tilde{\mathcal{F}}_{\tau}\right)^{\prime} h\right] d V
$$

which can easily be seen to vanish by linearizing (2.38).

By the formulas of Section 2

$$
\left(\nabla \tilde{\mathcal{F}}_{\tau}\right)_{i j}=-\Delta R_{i j}+(1+2 \tau) \nabla_{i} \nabla_{j} R-\left(\frac{1}{2}+2 \tau\right)(\Delta R) g_{i j}+\cdots,
$$

where "..." denotes terms which involve at most two derivatives of the metric. Linearizing this expression,

$$
\left(\nabla \tilde{\mathcal{F}}_{\tau}\right)^{\prime} h_{i j}=-\Delta R_{i j}^{\prime}+(1+2 \tau) \nabla_{i} \nabla_{j} R^{\prime}-\left(\frac{1}{2}+2 \tau\right)\left(\Delta R^{\prime}\right) g_{i j}+\cdots,
$$

where "..." now indicates terms which involve at most two derivatives of $h$. By standard formulas for the linearization of the Ricci and scalar curvatures,

$$
\begin{aligned}
R_{i j}^{\prime} & =-\frac{1}{2}\left[\Delta h_{i j}-\nabla_{i} \delta_{j} h-\nabla_{j} \delta_{j} h+\nabla_{i} \nabla_{j}(\operatorname{tr} h)\right]+\cdots, \\
R^{\prime} & =-\Delta(\operatorname{tr} h)+\delta^{2} h+\cdots .
\end{aligned}
$$

Consequently,

$$
\begin{aligned}
\left(\nabla \tilde{\mathcal{F}}_{\tau}\right)_{g}^{\prime} h_{i j}= & \frac{1}{2} \Delta^{2} h_{i j}-\frac{1}{2} \Delta\left[\nabla_{i} \delta_{j} h+\nabla_{j} \delta_{i} h\right]-\left(2 \tau+\frac{1}{2}\right) \nabla_{i} \nabla_{j}(\Delta \operatorname{tr} h) \\
& +(2 \tau+1) \nabla_{i} \nabla_{j}\left(\delta^{2} h\right)+\left(2 \tau+\frac{1}{2}\right)\left[\Delta^{2}(\operatorname{tr} h)-\Delta\left(\delta^{2} h\right)\right] g_{i j}+\cdots
\end{aligned}
$$

Also, a simple calculation gives

$$
\begin{gathered}
\frac{1}{2} \mathcal{K}_{g}\left[\square_{\mathcal{K}_{g}} \beta_{g} h\right]_{i j}=\frac{1}{2} \Delta[ \\
{\left[\nabla_{i} \delta_{j} h+\nabla_{j} \delta_{i} h\right]-\frac{2(n-1)}{n^{2}} \nabla_{i} \nabla_{j}(\Delta \operatorname{tr} h)+\frac{n-2}{n} \nabla_{i} \nabla_{j}\left(\delta^{2} h\right)} \\
+\frac{2(n-1)}{n^{3}} \Delta^{2}(\operatorname{tr} h) g_{i j}-\frac{2(n-1)}{n^{2}} \Delta\left(\delta^{2} h\right) g_{i j}+\cdots
\end{gathered}
$$

Therefore,

$$
\begin{aligned}
S h_{i j}= & \frac{1}{2} \Delta^{2} h_{i j}-\left[2 \tau+\frac{n^{2}+4 n-4}{2 n^{2}}\right] \nabla_{i} \nabla_{j}(\Delta \operatorname{tr} h)+2\left[\tau+\frac{n-1}{n}\right] \nabla_{i} \nabla_{j}\left(\delta^{2} h\right) \\
& +\left[2 \tau+\frac{n^{3}+4 n-4}{2 n^{3}}\right] \Delta^{2}(\operatorname{tr} h) g_{i j}-\left[2 \tau+\frac{n^{2}+4 n-4}{2 n^{2}}\right] \Delta\left(\delta^{2} h\right) g_{i j}+\cdots
\end{aligned}
$$


This implies the symbol $\left(\sigma_{\xi} S\right) h$ is given by

$$
\begin{aligned}
\left(\sigma_{\xi} S\right) h_{i j}= & \frac{1}{2}|\xi|^{4} h_{i j}-\left[2 \tau+\frac{n^{2}+4 n-4}{2 n^{2}}\right] \xi_{i} \xi_{j}|\xi|^{2}(\operatorname{tr} h)+2\left[\tau+\frac{n-1}{n}\right] \xi_{i} \xi_{j} h_{k \ell} \xi_{k} \xi_{\ell} \\
& +\left[2 \tau+\frac{n^{3}+4 n-4}{2 n^{3}}\right]|\xi|^{4}(\operatorname{tr} h) g_{i j}-\left[2 \tau+\frac{n^{2}+4 n-4}{2 n^{2}}\right]|\xi|^{2} h_{k \ell} \xi_{k} \xi_{\ell} g_{i j}
\end{aligned}
$$

To see that the symbol is non-degenerate, suppose $\left(\sigma_{\xi} S\right) h=0$ and $\xi \neq 0$. Then

$$
0=\frac{1}{|\xi|^{2}}\left(\sigma_{\xi} S\right) h_{i j} \xi_{i} \xi_{j}=\frac{2\left(n^{2}-1\right)}{n^{2}}\left[h_{k \ell} \xi_{k} \xi_{\ell}-\frac{1}{n}|\xi|^{2}(\operatorname{tr} h)\right]|\xi|^{2}
$$

hence

$$
h_{k \ell} \xi_{k} \xi_{\ell}=\frac{1}{n}|\xi|^{2}(\operatorname{tr} h) .
$$

Also, taking the trace of (2.56),

$$
\begin{aligned}
0=g^{i j}\left(\sigma_{\xi} S\right) h_{i j}= & {\left[2 \tau(n-1)+\frac{n}{2}\right]|\xi|^{4}(\operatorname{tr} h) } \\
& -\left[2 \tau(n-1)+\frac{n}{2}\right]|\xi|^{2} h_{k \ell} \xi_{k} \xi_{\ell} .
\end{aligned}
$$

In view of (2.58), this implies

$$
0=\frac{2(n-1)^{2}}{n}\left[\tau+\frac{n}{4(n-1)}\right]|\xi|^{4}(\operatorname{tr} h) .
$$

Since $\tau \neq-n / 4(n-1)$, we conclude that $\operatorname{tr} h=0$. As a consequence of this and (2.57), the symbol reduces to

$$
0=\left(\sigma_{\xi} S\right) h_{i j}=\frac{1}{2}|\xi|^{4} h_{i j}
$$

so that $h_{i j}=0$. It follows that the symbol is injective and $S$ is elliptic.

2.3. The Bach tensor. The Bach tensor in dimension four was introduced in Section 1.4. In this section, we study the local properties of the moduli space of Bach-flat metrics, in analogy with Section 2.2 above. Suppose $g$ is a Bach-flat metric, and let $S_{0}^{2}\left(T^{*} M\right)$ denote the space of trace-free (with respect to $g$ ) symmetric $(0,2)$-tensor fields. Consider

$$
P_{g}^{B} \theta=B(g+\theta)+\mathcal{K}_{g+\theta}\left(\square_{\mathcal{K}_{g}} \beta_{g} \theta\right)-\frac{1}{4} \operatorname{tr}_{g}\left\{B(g+\theta)+\mathcal{K}_{g+\theta}\left(\square_{\mathcal{K}_{g}} \beta_{g} \theta\right)\right\} g .
$$

The $g$-term ensures that $P_{g}^{B}: S_{0}^{2}\left(T^{*} M\right) \rightarrow S_{0}^{2}\left(T^{*} M\right)$.

Theorem 2.11. Assume $(M, g)$ is Bach-flat, and consider $P_{g}^{B}$ as a mapping from $C^{k+1, \alpha}$ to $C^{k-3, \alpha}$ for $k \geq 3$.

(i) The linearization of $P_{g}^{B}$ at $\theta=0$ is an elliptic operator.

(ii) If $\theta$ is sufficiently small and $P_{g}^{B}(\theta)=0$, then for each $p \in M$, there exists a locally defined function $u: U \rightarrow \mathbb{R}$, where $U$ is a neighborhood of $p$, such that the metric $e^{u}(g+\theta)$ is a smooth Riemannian metric and is Bach-flat. 
(iii) Conversely, if $g_{1}=g+\theta_{1}$ is a Bach-flat metric in a sufficiently small $C^{k+1, \alpha}$ neighborhood of $g(k \geq 3)$, then there exists a $C^{k+2, \alpha}$-diffeomorphism $\phi: M \rightarrow M$ and a function $u \in C^{k+1, \alpha}(M)$ such that

$$
e^{u} \phi^{*} g_{1}=g+\tilde{\theta}
$$

with $\tilde{\theta} \in S_{0}^{2}\left(T^{*} M\right)$ and

$$
P_{g}^{B}(\tilde{\theta})=0
$$

Proof. We will denote the linearized operator by $S_{g}^{B}$. The proof of ellipticity of $S_{g}^{B}$ is essentially the same as the proof in Theorem 2.7, since the Bach tensor is a multiple of the Euler-Lagrange equations of $\mathcal{F}_{-1 / 3}$.

For part $(i i)$, assume $P_{g}^{B}(\theta)=0$, and let $\tilde{g}=g+\theta$. Then

$$
0=B(\tilde{g})+\mathcal{K}_{\tilde{g}}\left(\square_{\mathcal{K}_{g}} \beta_{g} \theta\right)-\frac{1}{4} \operatorname{tr}_{g}\left\{B(\tilde{g})+\mathcal{K}_{\tilde{g}}\left(\square_{\mathcal{K}_{g}} \beta_{g} \theta\right)\right\} g
$$

Taking the trace with respect to $\tilde{g}$, we get

$$
0=-\frac{1}{4} \operatorname{tr}_{\tilde{g}}\left\{B(\tilde{g})+\mathcal{K}_{\tilde{g}}\left(\square_{\mathcal{K}_{g}} \beta_{g} \theta\right)\right\}\left(\operatorname{tr}_{\tilde{g}} g\right) .
$$

Since $\tilde{g}$ is a small perturbation of $g$, the trace of $g$ with respect to $\tilde{g}$ is non-vanishing, and it follows that

$$
\operatorname{tr}_{\tilde{g}}\left\{B(\tilde{g})+\mathcal{K}_{\tilde{g}}\left(\square_{\mathcal{K}_{g}} \beta_{g} \theta\right)\right\}=0 .
$$

Substituting this into (2.62) gives

$$
0=B(\tilde{g})+\mathcal{K}_{\tilde{g}}\left(\square_{\mathcal{K}_{g}} \beta_{g} \theta\right) .
$$

Pairing both sides of (2.64) with $B(\tilde{g})$ and integrating gives

$$
0=\int|B(\tilde{g})|_{\tilde{g}}^{2} d V_{\tilde{g}}+\int\left\langle B(\tilde{g}), \mathcal{K}_{\tilde{g}}\left(\square_{\mathcal{K}_{g}} \beta_{g} \theta\right)\right\rangle d V_{\tilde{g}}
$$

(Note that, as in the proof of Theorem 2.7. we should first mollify $\theta$, then use a limiting argument to show that the Bach tensor vanishes, but as the argument is identical we will omit the details.) Since

$$
\left(\left.\mathcal{K}\right|_{S_{0}^{2}\left(T^{*} M\right)}\right)^{*}=-2 \delta
$$

it follows that

$$
\begin{aligned}
\int\left\langle B(\tilde{g}), \mathcal{K}_{\tilde{g}}\left(\square_{\mathcal{K}_{g}} \beta_{g} \theta\right)\right\rangle d V_{\tilde{g}} & =\int\left\langle\left(\mathcal{K}_{\tilde{g}}\right)^{*} B(\tilde{g}), \mathcal{K}_{\tilde{g}}\left(\square_{\mathcal{K}_{g}} \beta_{g} \theta\right)\right\rangle d V_{\tilde{g}} \\
& =\int\left\langle-2 \delta_{\tilde{g}} B(\tilde{g}), \mathcal{K}_{\tilde{g}}\left(\square_{\mathcal{K}_{g}} \beta_{g} \theta\right)\right\rangle d V_{\tilde{g}}=0,
\end{aligned}
$$

since the Bach tensor is divergence-free. It follows from (2.65) that $B(\tilde{g})=0$.

To prove the local regularity in part $(i i)$, one can locally solve the negative Yamabe problem with Dirichlet boundary data, and the Bach-flat equation for the conformal metric becomes

$$
\Delta R i c=R c * R m
$$


and higher regularity follows using harmonic coordinates and a bootstrap argument as in part (ii) of Theorem 2.7, this was also proved in [TV05, Theorem 6.4].

To prove part $($ iii $)$, we use a slight variant of the method used to prove part $($ iii $)$ of Theorem 2.7. Suppose $g_{1}$ is a Bach-flat metric in a $C^{k+1, \alpha}$-neighborhood of $g$ :

$$
B\left(g_{1}\right)=0 .
$$

Let $\left\{\omega_{1}, \ldots, \omega_{\kappa}\right\}$ denote a basis of the space of conformal Killing forms with respect to $g$, and consider the map

$\mathcal{H}: C^{k+2, \alpha}(T M) \times C^{k+1, \alpha}(M) \times \mathbb{R}^{\kappa} \times C^{k+1, \alpha}\left(S^{2}\left(T^{*} M\right)\right) \rightarrow C^{k, \alpha}\left(T^{*} M\right) \times C^{k+1, \alpha}(M)$ given by

$$
\begin{aligned}
\mathcal{H}(X, u, v, \theta) & =\mathcal{H}_{\theta}(X, u, v) \\
& =\left(\beta_{g}\left[e^{u} \phi_{X, 1}^{*}(g+\theta)\right]+\sum_{i} v_{i} \omega_{i}, e^{u} \operatorname{tr}_{g}\left[\phi_{X, 1}^{*}(g+\theta)\right]-4\right),
\end{aligned}
$$

where $\phi_{X, 1}$ denotes the diffeomorphism obtained by following the flow generated by the vector field $X$ for unit time.

Linearizing $\mathcal{H}$ in $(X, u, v)$ at $(X, u, v, \theta)=(0,0,0,0)$, we find

$$
\begin{aligned}
\mathcal{H}_{0}^{\prime}(Y, u, a) & =\left.\frac{d}{d \epsilon}\left(\beta_{g}\left[e^{\epsilon u} \phi_{\epsilon Y, 1}^{*}(g)\right]+\sum_{i}\left(\epsilon a_{i}\right) \omega_{i}, e^{\epsilon u} \operatorname{tr}_{g}\left[\phi_{\epsilon Y, 1}^{*}(g)\right]-4\right)\right|_{\epsilon=0} \\
& =\left(\beta_{g}[u g]+\beta_{g} \mathcal{L}_{g} Y^{b}+\sum_{i} a_{i} \omega_{i}, 4 u+\operatorname{tr}_{g} \mathcal{L}_{g} Y^{b}\right) \\
& =\left(\square_{\mathcal{K}_{g}} Y^{b}+\sum_{i} a_{i} \omega_{i}, 4 u+2 \delta_{g} Y^{b}\right)
\end{aligned}
$$

where $Y^{b}$ is the dual one-form to $Y$. The adjoint map $\left(\mathcal{H}_{0}^{\prime}\right)^{*}: C^{m+2, \alpha}\left(T^{*} M\right) \times$ $C^{m+1, \alpha}(M) \rightarrow C^{m, \alpha}(T M) \times \mathbb{R}^{\kappa} \times C^{m+1, \alpha}(M)$ is given by

$$
\left(\mathcal{H}_{0}^{\prime}\right)^{*}(\eta, f)=\left(\left(\square_{\mathcal{K}_{g}} \eta\right)^{\sharp}-2 \nabla f, 4 f, \int\left\langle\eta, \omega_{i}\right\rangle d V\right),
$$

where $\left(\square_{\mathcal{L}_{g}} \omega\right)^{\sharp}$ is the vector field dual to $\square_{\mathcal{L}_{g}} \eta$. It follows that if $(\eta, f) \in \operatorname{ker}\left(\mathcal{H}_{0}^{\prime}\right)^{*}$, then $f=0$ and

$$
\begin{aligned}
\square_{\mathcal{K}_{g}} \eta & =0, \\
\int\left\langle\eta, \omega_{i}\right\rangle d V & =0, \quad 1 \leq i \leq \kappa .
\end{aligned}
$$

Therefore, $\eta=0$ and the map $\mathcal{H}_{0}^{\prime}$ is surjective. Consequently, by the implicit function theorem, given $\theta_{1} \in C^{k+1, \alpha}\left(S^{2}\left(T^{*} M\right)\right)$ small enough we can solve the equation

$$
\mathcal{H}_{\theta_{1}}(X, u, v)=(0,0)
$$


i.e., there is a vector field $X \in C^{k+2, \alpha}(T M)$, a function $u \in C^{k+1, \alpha}(M)$, and a vector $v \in \mathbb{R}^{\kappa}$ such that

$$
\begin{aligned}
\beta_{g}\left[e^{u} \phi_{X, 1}^{*}(g+\theta)\right]+\sum_{i} v_{i} \omega_{i} & =0, \\
e^{u} \operatorname{tr}_{g}\left[\phi_{X, 1}^{*}(g+\theta)\right]-4 & =0 .
\end{aligned}
$$

As in the proof of Lemma 2.10, the first equation implies

$$
\beta_{g}\left[e^{u} \phi^{*} g_{1}\right]=0
$$

where $\phi=\phi_{X, 1}$. If we write

$$
e^{u} \phi^{*} g_{1}=g+\tilde{\theta}
$$

then the second equation in (2.70) implies

$$
\operatorname{tr}_{g} \tilde{\theta}=\operatorname{tr}_{g}\left[e^{u} \phi^{*} g_{1}-g\right]=\operatorname{tr}_{g}\left[e^{u} \phi_{X, 1}^{*}\left(g_{1}\right)\right]-4=0
$$

hence $\tilde{\theta} \in S_{0}^{2}\left(T^{*} M\right)$.

It follows that the gauge-fixing term in (2.59) vanishes:

$$
\mathcal{K}_{g+\tilde{\theta}}\left(\square_{\mathcal{K}_{g}} \beta_{g} \tilde{\theta}\right)=0 .
$$

Therefore,

$$
\begin{aligned}
P_{g}^{B}(\tilde{\theta}) & =B(g+\tilde{\theta})+\mathcal{K}_{g+\tilde{\theta}}\left(\square_{\mathcal{K}_{g}} \beta_{g} \tilde{\theta}\right)-\frac{1}{4} \operatorname{tr}_{g}\left\{B(g+\tilde{\theta})+\mathcal{K}_{g+\tilde{\theta}}\left(\square_{\mathcal{K}_{g}} \beta_{g} \tilde{\theta}\right)\right\} g \\
& =B(g+\tilde{\theta})-\frac{1}{4} \operatorname{tr}_{g}\{B(g+\tilde{\theta})\} g=B\left(e^{u} \phi^{*} g_{1}\right)-\frac{1}{4} \operatorname{tr}_{g}\left\{B\left(e^{u} \phi^{*} g_{1}\right)\right\} g \\
& =e^{-u} B\left(\phi^{*} g_{1}\right)-\frac{1}{4} \operatorname{tr}_{g}\left\{e^{-u} B\left(\phi^{*} g_{1}\right)\right\} g \\
& =e^{-u} \phi^{*}\left(B\left(g_{1}\right)\right)-\frac{1}{4} \operatorname{tr}_{g}\left\{e^{-u} \phi^{*}\left(B\left(g_{1}\right)\right)\right\} g=0,
\end{aligned}
$$

verifying (2.61).

2.4. The Kuranishi Map. In view of our preceding results, it is possible to describe the moduli space of critical metrics near $g$ as the zeroes of a Fredholm map. In fact, using the Implicit Function Theorem the moduli space can be described as the zero set of a finite-dimensional map called the Kuranishi map. To make this more precise, we define $H_{\tau}^{1}$ to be the kernel of the linearization of $P_{g}$ :

$$
H_{\tau}^{1}=H_{\tau}^{1}(M, g)=\left\{h \in C^{4, \alpha}\left(\bar{S}_{0}^{2}\left(T^{*} M\right)\right) \mid S_{g} h=0\right\} .
$$

In the case $n=4$ and $\tau=-1 / 3$ (the Bach tensor), we restrict to traceless tensors:

$$
H_{-1 / 3}^{1}=H_{\tau}^{1}(M, g)=\left\{h \in C^{4, \alpha}\left(S_{0}^{2}\left(T^{*} M\right)\right) \mid S_{g}^{B} h=0\right\} .
$$

Since $S_{g}$ is elliptic, $H_{\tau}^{1}$ is finite dimensional. Moreover, we can characterize $H_{\tau}^{1}$ in terms of the linearization of $\nabla \tilde{\mathcal{F}}_{\tau}$ : 
Lemma 2.12. We have

$$
H_{\tau}^{1}=\left\{h \in C^{\infty}\left(\bar{S}_{0}^{2}\left(T^{*} M\right)\right) \mid\left(\nabla \tilde{\mathcal{F}}_{\tau}\right)_{g}^{\prime} h=0, \beta_{g} h=0\right\} .
$$

In the case $n=4$ and $\tau=-1 / 3$,

$$
H_{-1 / 3}^{1}=\left\{h \in C^{\infty}\left(S_{0}^{2}\left(T^{*} M\right)\right) \mid B_{g}^{\prime} h=0, \delta_{g} h=0\right\} .
$$

Proof. Smoothness follows from ellipticity of $S_{g}$. Recall from (2.52) that

$$
S_{g} h=\left.\frac{d}{d s} P_{g}(g+s h)\right|_{s=0}=\left(\nabla \tilde{\mathcal{F}}_{\tau}\right)_{g}^{\prime}(h)+\frac{1}{2} \mathcal{K}_{g}\left[\square_{\mathcal{K}_{g}} \beta_{g} h\right] .
$$

Clearly, it suffices to show that if $S_{g} h=0$, then $\beta_{g} h=0$. To see this, we first note that given a path of metrics $g(s),-\epsilon<s<\epsilon$, with $g(0)=g$, then

$$
\delta_{g(s)}\left[\left(\nabla \tilde{\mathcal{F}}_{\tau}\right)_{g(s)}\right]=0,
$$

since the gradient of a Riemannian functional is divergence-free. If $g^{\prime}(0)=h$, then linearizing this identity at $s=0$ gives

$$
\delta_{g}\left[\left(\nabla \tilde{\mathcal{F}}_{\tau}\right)_{g}^{\prime} h\right]=0 .
$$

Therefore, if $S_{g} h=0$, it follows that

$$
0=\delta_{g} S_{g} h=\delta_{g}\left[\left(\nabla \tilde{\mathcal{F}}_{\tau}\right)_{g}^{\prime} h\right]+\frac{1}{2} \delta_{g} \mathcal{K}_{g}\left[\square_{\mathcal{K}_{g}} \beta_{g} h\right]=\frac{1}{2} \square_{\mathcal{K}_{g}}^{2} \beta_{g} h .
$$

Consequently, $\mathcal{K}_{g} \beta_{g} h=0$. But

$$
\left\|\beta_{g} h\right\|_{L^{2}}^{2}=\left\langle\beta_{g} h, \beta_{g} h\right\rangle_{L^{2}}=\left\langle h, \beta_{g}^{*} \beta_{g} h\right\rangle_{L^{2}}=\left\langle h,-\frac{1}{2} \mathcal{K}_{g} \beta_{g} h\right\rangle_{L^{2}}=0 .
$$

The proof for the Bach tensor is identical.

We will now show there is a well defined mapping $\Psi: H_{\tau}^{1} \rightarrow H_{\tau}^{2}$, called the Kuranishi map, for which the moduli space is locally given by $\Psi^{-1}(0)$, where $H_{\tau}^{2}$ is the cokernel of the linearized operator. (Note that since $S_{g}$ is self-adjoint, on a compact manifold we have $H_{\tau}^{2}=H_{\tau}^{1}$.) To do this, we need to prove a technical lemma about the structure of the nonlinear terms in the Taylor expansion of $P=P_{g}$ :

Lemma 2.13. There exists a constant $C$ such that if we write

$$
P_{g}(h)=P_{g}(0)+S_{g} h+Q(h),
$$

then for $h_{1}, h_{2} \in C^{4, \alpha}$ of sufficiently small norm,

$$
\left\|Q\left(h_{1}\right)-Q\left(h_{2}\right)\right\|_{C^{\alpha}} \leq C\left(\left\|h_{1}\right\|_{C^{4, \alpha}}+\left\|h_{2}\right\|_{C^{4, \alpha}}\right) \cdot\left\|h_{1}-h_{2}\right\|_{C^{4, \alpha}} .
$$

Proof. Since the proof involves a rather lengthy calculation we begin with a brief overview. The tensor $\nabla \tilde{\mathcal{F}}_{\tau}$ can be schematically expressed as

$$
\nabla \tilde{\mathcal{F}}_{\tau}(g)=g * g^{-1} * g^{-1} * \nabla_{g}^{2} R m_{g}+g * g^{-1} * g^{-1} * R m_{g} * R m_{g},
$$

where $R m_{g}$ denotes the curvature tensor of $g, g^{-1} * \cdots * g^{-1} * A * B$ denotes any linear combination of terms involving contractions of the tensor product $A \otimes B$, and 
$g^{-1} * \cdots * g^{-1} * \nabla_{g}^{k} * A$ denotes linear combinations of contractions of the $k$-th iterated covariant derivative of $A$. Since the mapping $P$ is defined by

$$
P_{g}(h)=\nabla \tilde{\mathcal{F}}_{\tau}(g+h)+\frac{1}{2} \mathcal{K}_{g+h}\left[\square_{\mathcal{K}_{g}} \beta_{g} \theta\right]-\gamma(h) \cdot g,
$$

the first step in proving the estimates is to analyze the curvature term

$$
\begin{aligned}
\nabla \tilde{\mathcal{F}}_{\tau}(g+h)= & (g+h) *(g+h)^{-1} *(g+h)^{-1} * \nabla_{g+h}^{2} R m_{g+h} \\
& +(g+h) *(g+h)^{-1} *(g+h)^{-1} * R m_{g+h} * R m_{g+h} .
\end{aligned}
$$

Proceeding as in AV12, Proposition 3.1], the starting point is the formula

$$
\Gamma(g+h)_{i j}^{k}=\Gamma(g)_{i j}^{k}+\frac{1}{2}(g+h)^{k m}\left\{\nabla_{j} h_{i m}+\nabla_{i} h_{j m}-\nabla_{m} h_{i j}\right\}
$$

where $\Gamma(\cdot)$ denotes the Christoffel symbols of a metric. Using this formula and the notation introduced above, we can express the covariant derivative with respect to the metric $g+h$ as

$$
\nabla_{g+h} T=\nabla_{g} T+(g+h)^{-1} * \nabla_{g} h * T
$$

where $T$ is any tensor field. Also, by the standard formula for the $(1,3)$-curvature tensor in terms of the Christoffel symbols we have

$$
R m_{g+h}=R m_{g}+(g+h)^{-1} * \nabla^{2} h+(g+h)^{-2} * \nabla h * \nabla h .
$$

In the following, any covariant derivative without a subscript will mean with respect to the fixed metric $g$.

Taking two covariant derivatives $\nabla_{g+h}$ of $R m_{g+h}$ and repeatedly using (2.80), we obtain

$$
\begin{aligned}
(g & +h) *\left\{(g+h)^{-2} * \nabla_{g+h}^{2} R m_{g+h}+(g+h)^{-2} * R m_{g+h} * R m_{g+h}\right\} \\
= & (g+h) *\left\{(g+h)^{-2} * \nabla^{2} R m_{g}+(g+h)^{-2} * R m_{g} * R m_{g}\right. \\
& +(g+h)^{-3} * \nabla R m_{g} * \nabla h+(g+h)^{-3} * R m_{g} * \nabla^{2} h \\
& +(g+h)^{-4} * R m_{g} * \nabla h * \nabla h+(g+h)^{-3} * \nabla^{4} h \\
& +(g+h)^{-4} * \nabla^{3} h * \nabla h+(g+h)^{-4} * \nabla^{2} h * \nabla^{2} h \\
& \left.+(g+h)^{-5} * \nabla^{2} h * \nabla h * \nabla h+(g+h)^{-6} * \nabla h * \nabla h * \nabla h * \nabla h\right\} .
\end{aligned}
$$


Taking into account the term $\gamma(h) g$, and the gauge-fixing term in (2.77), we obtain

$$
\begin{aligned}
P_{g}(h)= & (g+h) *\left\{(g+h)^{-2} * \nabla^{2} R m_{g}+(g+h)^{-2} * R m_{g} * R m_{g}\right. \\
& +(g+h)^{-3} * \nabla R m_{g} * \nabla h+(g+h)^{-3} * R m_{g} * \nabla^{2} h \\
& +(g+h)^{-4} * R m_{g} * \nabla h * \nabla h+(g+h)^{-3} * \nabla^{4} h \\
& +(g+h)^{-1} * g^{-3} * g * \nabla^{4} h+(g+h)^{-4} * \nabla^{3} h * \nabla h \\
& +(g+h)^{-2} * g * g^{-3} * \nabla^{3} h * \nabla h+(g+h)^{-4} * \nabla^{2} h * \nabla^{2} h \\
& +(g+h)^{-5} * \nabla^{2} h * \nabla h * \nabla h \\
& \left.+(g+h)^{-6} * \nabla h * \nabla h * \nabla h * \nabla h\right\}+\int\{\cdots\} d V,
\end{aligned}
$$

where $\{\cdots\}$ denotes the terms in (2.82).

Since we are trying to estimate the remainder terms in the Taylor expansion of $P(h)$, we want to write the above expression in terms of its linearization; i.e.,

$$
\begin{aligned}
P_{g}(h) & =P_{g}(0)+S_{g} h+\cdots \\
& =\nabla \tilde{\mathcal{F}}_{\tau}(g)+S_{g} h+\cdots
\end{aligned}
$$

To do this, we use the identity (which holds for $h$ small and all integers $k \geq 1$ )

$$
(g+h)^{-k}-g^{-k}=g^{-k-1} * h+r_{k}(h),
$$

where the remainder satisfies

$$
\left|r_{k}\left(h_{1}\right)-r_{k}\left(h_{2}\right)\right| \leq C_{k}(g)\left(\left|h_{1}\right|+\left|h_{2}\right|\right)\left|h_{1}-h_{2}\right|,
$$

with a similar estimate for the Hölder norm.

Next, we substitute (2.84) into each term of (2.83) involving a power of $(g+h)^{-1}$, then collect all terms which are zeroth order in $h$ (which combine to give $P_{g}(0)$ ), those which are linear in $h$ (which combine to give $S h$ ), and those which are higher order in $h$.

For example, consider the term $(g+h) *(g+h)^{-3} * \nabla^{4} h$. Applying (2.84), we may write this as

$$
\begin{aligned}
(g+h) *(g+h)^{-3} * \nabla^{4} h & =g * g^{-3} * \nabla^{4} h+g * g^{-4} * h * \nabla^{4} h+g * g^{-4} * r_{3}(h) * \nabla^{4} h \\
& +g^{-3} * h * \nabla^{4} h+g^{-4} * h * h * \nabla^{4} h+g^{-4} * r_{3}(h) * \nabla^{4} h .
\end{aligned}
$$

It is clear that only the first term on the right hand side contributes to the linearization, hence we need to estimate the rest of the terms. Consider the next term on the right-hand side of (2.86):

$$
T(h)=g * g^{-4} * h * \nabla^{4} h
$$

Then

$$
\begin{aligned}
T\left(h_{1}\right)-T\left(h_{2}\right) & =g * g^{-4} * h_{1} * \nabla^{4} h_{1}-g * g^{-4} * h_{2} * \nabla^{4} h_{2} \\
& =g * g^{-4} *\left(h_{1}-h_{2}\right) * \nabla^{4} h_{1}+g * g^{-4} * h_{2} * \nabla^{4}\left(h_{1}-h_{2}\right),
\end{aligned}
$$


hence

$$
\left|T\left(h_{1}\right)-T\left(h_{2}\right)\right| \leq\left|h_{1}-h_{2}\right|\left|\nabla^{4} h_{1}\right|+\left|h_{2}\right|\left|\nabla^{4}\left(h_{1}-h_{2}\right)\right|
$$

and it follows that

$$
\begin{aligned}
\left\|T\left(h_{1}\right)-T\left(h_{2}\right)\right\|_{C^{0}} & \leq\left\{\left\|h_{1}-h_{2}\right\|_{C^{0}}\left\|h_{1}\right\|_{C^{4}}+\left\|h_{2}\right\|_{C^{0}}\left\|h_{1}-h_{2}\right\|_{C^{4}}\right\} \\
& \leq C\left(\left\|h_{1}\right\|_{C^{4}}+\left\|h_{2}\right\|_{C^{4}}\right) \cdot\left\|h_{1}-h_{2}\right\|_{C^{4}} .
\end{aligned}
$$

Using (2.85), the rest of the terms on the right hand side of (2.86) can be estimated similarly. Estimating difference quotients gives the required Holder estimate. Analyzing each term in (2.83) in a similar fashion, (2.75) follows..

The main consequence of this formula is the following:

Corollary 2.14. The zero set of $P_{g}$ is locally modeled on the zero set of a finitedimensional map

$$
\Psi: H_{\tau}^{1} \rightarrow H_{\tau}^{2} \simeq H_{\tau}^{1}
$$

Consequently, if $g$ is infinitesimally rigid, then $g$ is rigid. In the case $n=4$ and $\tau=-1 / 3$, infinitesimal Bach-rigidity implies Bach-rigidity.

In general, the map $\Psi$ is equivariant with respect to the isometry group $\mathcal{I}(g)$. If $\mathcal{C}(g)=\mathcal{I}(g)$, where $\mathcal{C}(g)$ is the conformal automorphism group of $g$, then the moduli space of solutions, up to diffeomorphism and scaling, is given by the space of orbits $\Psi^{-1}(0) / \mathcal{I}(g)$.

Proof. The existence of the map $\Psi$ follows from Lemma 2.13 , together with a fixedpoint argument as in [RS05, Proposition 4.2.1]. The argument above identified the zero set of $P_{g}$ with the moduli space of solutions modulo diffeomorphism and scaling, up to uniqueness of the gauge $\beta_{g}(\tilde{\theta})=0$ found in Lemma 2.10. This gauge is unique up to the action of the conformal group, since the kernel of the linearization $\mathcal{N}_{0}^{\prime}$ is the space of conformal Killing fields. Under the assumption that $\mathcal{C}(g)=\mathcal{I}(g)$, the gauge is therefore unique up to the action of the isometry group. The same argument holds for $P_{g}^{B}$ in the case $n=4, \tau=-1 / 3$.

Remark 2.15. It might seem more natural to use the gauge $\delta_{g} \tilde{\theta}=0$ in Lemma 2.10 , instead of the gauge $\beta_{g} \tilde{\theta}=0$. With a suitable modification of the map $P_{g}$, this approach indeed works, thus obviating the assumtion that $\mathcal{C}(g)=\mathcal{I}(g)$ in the second part of Corollary 2.14, However, it is substantially more efficient to do compuations in the $\beta_{g} \tilde{\theta}=0$ gauge, so we prefer to work in this gauge. There is no loss of generality for Einstein metrics, since for these it is true that $\mathcal{C}(g)=\mathcal{I}(g)$, with the only exceptional case being that of the round sphere.

\section{Second variation at an Einstein metric}

We begin this section by expressing the second variation of the volume normalized functional in terms of the un-normalized functional. In the latter part of the section, we will compute the full second variation of the un-normalized functional. These will then be combined later in Sections 3.3 and 3.4 . 
3.1. Normalized functional. As before, we let $\mathcal{F}_{\tau}$ denote any quadratic curvature functional, and $\tilde{\mathcal{F}}_{\tau}$ denote the associated volume normalized functional.

Lemma 3.1. If $g$ is critical for $\tilde{\mathcal{F}}_{\tau}$ then

$$
\left(\tilde{\mathcal{F}}_{\tau}\left[g_{s}\right]\right)^{\prime \prime}=-p(p+1)(V o l)^{p-2}\left((V o l)^{\prime}\right)^{2} \cdot \mathcal{F}_{\tau}+p(V o l)^{p-1}(V o l)^{\prime \prime} \cdot \mathcal{F}_{\tau}+(V o l)^{p} \cdot\left(\mathcal{F}_{\tau}\right)^{\prime \prime} .
$$

Proof. Differentiating (2.16), we obtain

$$
\begin{aligned}
\left(\tilde{\mathcal{F}}_{\tau}\left[g_{s}\right]\right)^{\prime \prime} & =p(p-1)(V o l)^{p-2}\left(V o l^{\prime}\right)\left(V o l^{\prime}\right) \cdot \mathcal{F}_{\tau}+p(V o l)^{p-1}(V o l)^{\prime \prime} \cdot \mathcal{F}_{\tau} \\
& +2 p(V o l)^{p-1}(V o l)^{\prime} \cdot\left(\mathcal{F}_{\tau}\left[g_{s}\right]\right)^{\prime}+(V o l)^{p}\left(\mathcal{F}_{\tau}\left[g_{s}\right]\right)^{\prime \prime} .
\end{aligned}
$$

The result follows by using (2.14) on the third term on the right hand side and combining with the first term to give

$$
\begin{aligned}
& p(p-1)(V o l)^{p-2}\left(V o l^{\prime}\right)\left(V o l^{\prime}\right) \cdot \mathcal{F}_{\tau}+2 p(V o l)^{p-1}(V o l)^{\prime}\left(-p(V o l)^{-1}(V o l)^{\prime} \cdot \mathcal{F}_{\tau}\right) \\
& =-p(p+1)(V o l)^{p-2}\left(\left(V o l^{\prime}\right)\right)^{2} \cdot \mathcal{F}_{\tau} .
\end{aligned}
$$

Next, we compute the second derivative of the unnormalized functional $\mathcal{F}_{\tau}$.

Lemma 3.2. If $\nabla \mathcal{F}_{\tau}=c \cdot g$, then

$$
\begin{aligned}
\frac{d^{2}}{d s^{2}}\left(\mathcal{F}_{\tau}\left[g_{s}\right]\right) & =\int\left\langle\left(\nabla \mathcal{F}_{\tau}\right)^{\prime}\left(g_{s}\right) g_{s}^{\prime}, g_{s}^{\prime}\right\rangle d V \\
& -2 c \int\left|g_{s}^{\prime}\right|^{2} d V+c \int\left(\operatorname{tr}_{g_{s}} g_{s}^{\prime \prime}\right) d V+\frac{c}{2} \int\left(\operatorname{tr}_{g_{s}} g_{s}^{\prime}\right)^{2} d V
\end{aligned}
$$

Proof. For any path of metrics $g_{s}$, we have

$$
\frac{d}{d s}\left(\mathcal{F}_{\tau}\left[g_{s}\right]\right)=\int\left\langle\nabla \mathcal{F}_{\tau}\left(g_{s}\right), g_{s}^{\prime}\right\rangle d V
$$

where $\nabla \mathcal{F}_{\tau}$ are the Euler-Lagrange equations. The integrand is locally

$$
g^{i p} g^{j q}\left(\nabla \mathcal{F}_{\tau}\left(g_{s}\right)\right)_{i j}\left(g_{s}^{\prime}\right)_{p q} d V .
$$

Taking another derivative,

$$
\begin{aligned}
\frac{d^{2}}{d s^{2}}\left(\mathcal{F}_{\tau}\left[g_{s}\right]\right) & =-2 \int\left(g_{s}^{\prime}\right)^{i p} g^{j q}\left(\nabla \mathcal{F}_{\tau}\left(g_{s}\right)_{i j}\left(g_{s}^{\prime}\right)_{p q} d V+\int\left\langle\left(\nabla \mathcal{F}_{\tau}\right)^{\prime}\left(g_{s}\right) g_{s}^{\prime}, g_{s}^{\prime}\right\rangle d V\right. \\
& +\int\left\langle\nabla \mathcal{F}_{\tau}\left(g_{s}\right), g_{s}^{\prime \prime}\right\rangle d V+\int\left\langle\nabla \mathcal{F}_{\tau}\left(g_{s}\right), g_{s}^{\prime}\right\rangle(d V)^{\prime} .
\end{aligned}
$$

If $\nabla \mathcal{F}_{\tau}\left(g_{s}\right)=c g$, then this simplifies to (3.4).

The next result gives the relation between the second variation of the un-normalized and normalized functionals: 
Proposition 3.3. If $g$ is Einstein with $\nabla \mathcal{F}_{\tau}=c \cdot g$, then

$$
\begin{aligned}
\left(\tilde{\mathcal{F}}_{\tau}\left[g_{s}\right]\right)^{\prime \prime} & =-p(p+1)(V o l)^{p-2}\left((V o l)^{\prime}\right)^{2} \cdot \mathcal{F}_{\tau} \\
& +(V o l)^{p}\left\{-c \int_{M}\left|g_{s}^{\prime}\right|^{2} d V+\int\left\langle\left(\nabla \mathcal{F}_{\tau}\right)^{\prime}\left(g_{s}\right) g_{s}^{\prime}, g_{s}^{\prime}\right\rangle d V\right\} .
\end{aligned}
$$

In particular, if $\left(\nabla \mathcal{F}_{\tau}\right)^{\prime}$ and $\left(\nabla \tilde{\mathcal{F}}_{\tau}\right)^{\prime}$ denote the linearized Euler-Lagrange equations of $\mathcal{F}_{\tau}$ and $\tilde{\mathcal{F}}_{\tau}$ respectively, then

$$
\left(\nabla \tilde{\mathcal{F}}_{\tau}\right)^{\prime} h=\left(\nabla \mathcal{F}_{\tau}\right)^{\prime} h-c h .
$$

Proof. Substituting (3.4) into (3.1), and using (2.13), we have

$$
\begin{aligned}
\left(\tilde{\mathcal{F}}_{\tau}\left[g_{s}\right]\right)^{\prime \prime} & =-p(p+1)(V o l)^{p-2}\left((V o l)^{\prime}\right)^{2} \cdot \mathcal{F}_{\tau}+p(V o l)^{p-1}(V o l)^{\prime \prime} \cdot \mathcal{F}_{\tau}+(V o l)^{p} \cdot\left(\mathcal{F}_{\tau}\right)^{\prime \prime} \\
& =-p(p+1)(V o l)^{p-2}\left((V o l)^{\prime}\right)^{2} \cdot \mathcal{F}_{\tau}+(V o l)^{p}\left(\int\left\langle\left(\nabla \mathcal{F}_{\tau}\right)^{\prime}\left(g_{s}\right) g_{s}^{\prime}, g_{s}^{\prime}\right\rangle d V\right) \\
& +p(V o l)^{p-1} \cdot \mathcal{F}_{\tau} \cdot\left(-\frac{1}{2} \int_{M}\left|g_{s}^{\prime}\right|^{2} d V+\frac{1}{2} \int_{M}\left(\operatorname{tr}_{g_{s}} g_{s}^{\prime \prime}\right) d V+\frac{1}{4} \int\left(\operatorname{tr}_{g_{s}} g_{s}^{\prime}\right)^{2} d V\right) \\
& +c(V o l)^{p}\left(-2 \int\left|g_{s}^{\prime}\right|^{2} d V+\int\left(\operatorname{tr}_{g_{s}} g_{s}^{\prime \prime}\right) d V+\frac{1}{2} \int\left(\operatorname{tr}_{g_{s}} g_{s}^{\prime}\right)^{2} d V\right) .
\end{aligned}
$$

From (2.15), we have that

$$
c=-\frac{p}{2}(V o l)^{-1} \cdot \mathcal{F}_{\tau},
$$

and substituting this into the above yields

$$
\begin{aligned}
\left(\tilde{\mathcal{F}}_{\tau}\left[g_{s}\right]\right)^{\prime \prime} & =-p(p+1)(V o l)^{p-2}\left((V o l)^{\prime}\right)^{2} \cdot \mathcal{F}_{\tau}+(V o l)^{p}\left(\int\left\langle\left(\nabla \mathcal{F}_{\tau}\right)^{\prime}\left(g_{s}\right) g_{s}^{\prime}, g_{s}^{\prime}\right\rangle d V\right) \\
& +p(V o l)^{p-1} \cdot \mathcal{F}_{\tau} \cdot\left(-\frac{1}{2} \int_{M}\left|g_{s}^{\prime}\right|^{2} d V+\frac{1}{2} \int_{M}\left(\operatorname{tr}_{g_{s}} g_{s}^{\prime \prime}\right) d V+\frac{1}{4} \int\left(\operatorname{tr}_{g_{s}} g_{s}^{\prime}\right)^{2} d V\right) \\
& -\frac{p}{2}(V o l)^{p-1} \cdot \mathcal{F}_{\tau} \cdot\left(-2 \int\left|g_{s}^{\prime}\right|^{2} d V+\int\left(\operatorname{tr}_{g_{s}} g_{s}^{\prime \prime}\right) d V+\frac{1}{2} \int\left(\operatorname{tr}_{g_{s}} g_{s}^{\prime}\right)^{2} d V\right),
\end{aligned}
$$

whichs yields the claimed formula.

Remark 3.4. At this point it is important to make a remark about the use of the terms second variation, linearized Euler-Lagrange equation, and Jacobi operator, and the amount of regularity necessary for these to be well-defined.

First, the $L^{2}$-gradient of $\tilde{\mathcal{F}}_{\tau}$ calculated in Section 2 is clearly fourth order in the metric, since it is obtained by integrating by parts. Consequently, the linearized Euler-Lagrange equation is a fourth-order operator on tensor fields. We also refer to this operator as the Jacobi operator. The second variation can be expressed as a bilinear form on symmetric tensor fields, as in (3.12) above.

Since $\tilde{\mathcal{F}}_{\tau}$ only involves two derivatives of the metric, the second variation is actually second order in the infinitesimal variation. Therefore, although we will write 
$\int\langle J h, h\rangle d V$ to denote the second variation (where $J$ is the Jacobi operator), this expression actually makes sense as long as $h \in C^{2}\left(S^{2}\left(T^{*} M\right)\right)$.

3.2. Jacobi operator. In this subsection, we will compute an expression for the linearized Euler-Lagrange equation of $\mathcal{F}_{\tau}$ for an arbitrary variation $h$ of an Einstein metric; i.e., the operator $\left(\nabla \mathcal{F}_{\tau}\right)^{\prime} h$. Subsequently, in Sections 3.3 and 3.4 we will examine the action of this operator on transverse-traceless tensors and pure trace tensors.

The first step is to linearize the hessian of the Ricci tensor at an Einstein metric:

Proposition 3.5. If $g$ is Einstein, satisfying Ric $=\frac{R}{n} g$, then with $h=g^{\prime}$, then

$$
\left(\nabla_{l} \nabla_{j} R_{k p}\right)^{\prime}=\nabla_{l} \nabla_{j}\left(R_{k p}\right)^{\prime}-\frac{R}{n} \cdot \nabla_{l} \nabla_{j} h_{k p} .
$$

Proof. We write out

$$
\nabla_{l}\left(\nabla_{j} R_{k p}\right)=\partial_{l}\left(\nabla_{j} R_{k p}\right)-\nabla_{q} R_{k p} \Gamma_{l j}^{q}-\nabla_{j} R_{q p} \Gamma_{l k}^{q}-\nabla_{j} R_{k q} \Gamma_{l p}^{q},
$$

and

$$
\nabla_{i} R_{j k}=\partial_{i} R_{j k}-R_{p k} \Gamma_{i j}^{p}-R_{j p} \Gamma_{i k}^{p} .
$$

Next we linearize, with $g^{\prime}=h$. We use tensorality to simply toss out terms which have Christoffel symbols, and replace derivatives with covariant derivatives to obtain

$$
\begin{aligned}
\left(\nabla_{l} \nabla_{j} R_{k p}\right)^{\prime} & =\nabla_{l} \nabla_{j}\left(R_{k p}\right)^{\prime}-R_{q p} \nabla_{l}\left(\Gamma_{j k}^{q}\right)^{\prime}-R_{k q} \nabla_{l}\left(\Gamma_{j p}^{q}\right)^{\prime} \\
& -\nabla_{q} R_{k p}\left(\Gamma_{l j}^{q}\right)^{\prime}-\nabla_{j} R_{q p}\left(\Gamma_{l k}^{q}\right)^{\prime}-\nabla_{j} R_{k q}\left(\Gamma_{l p}^{q}\right)^{\prime} .
\end{aligned}
$$

Since Ricci is parallel, the last three terms vanish, and using the formula

$$
\left(\Gamma_{i j}^{k}\right)^{\prime}=\frac{1}{2} g^{k l}\left(\nabla_{i} h_{j l}+\nabla_{j} h_{i l}-\nabla_{l} h_{i j}\right)
$$

we obtain

$$
\begin{aligned}
& \left(\nabla_{l} \nabla_{j} R_{k p}\right)^{\prime}=\nabla_{l} \nabla_{j}\left(R_{k p}\right)^{\prime}-R_{q p} \nabla_{l}\left(\frac{1}{2} g^{q m}\left(\nabla_{j} h_{k m}+\nabla_{k} h_{j m}-\nabla_{m} h_{j k}\right)\right) \\
& -R_{k q} \nabla_{l}\left(\frac{1}{2} g^{q m}\left(\nabla_{j} h_{p m}+\nabla_{p} h_{j m}-\nabla_{m} h_{j p}\right)\right)=\nabla_{l} \nabla_{j}\left(R_{k p}\right)^{\prime}-\frac{R}{n} \cdot \nabla_{l} \nabla_{j} h_{k p} .
\end{aligned}
$$

With these formulas we can linearize the Euler-Lagrange equations of $\mathcal{F}_{0}$ :

Theorem 3.6. If $g$ is Einstein, then

$$
\begin{aligned}
\left(\nabla \mathcal{F}_{0}\right)_{p q}^{\prime} & =-\Delta(R i c)_{p q}^{\prime}+\frac{R}{n} \Delta h_{p q}-2\left(\frac{R}{n}\left(\operatorname{Ric}_{p q}\right)^{\prime}-\frac{R}{n} R_{p k q l} h^{k l}+R_{p k q l} g^{k i} g^{l j}\left(\operatorname{Ric}^{\prime}\right)_{i j}\right) \\
& +\nabla_{p q}^{2}\left(\operatorname{tr}_{g} \operatorname{Ric}^{\prime}\right)-\frac{R}{n} \nabla_{p q}^{2}\left(\operatorname{tr}_{g} h\right)-\frac{1}{2}\left(\Delta\left(\operatorname{tr}_{g} \operatorname{Ric}^{\prime}\right)-\frac{R}{n} \Delta\left(\operatorname{tr}_{g} h\right)\right) g_{p q} \\
& +\left(-\frac{R^{2}}{n^{2}} \operatorname{tr}_{g} h+\frac{R}{n} \operatorname{tr}_{g}\left(\operatorname{Ric}^{\prime}\right)\right) g_{p q}+\frac{R^{2}}{2 n} h_{p q} .
\end{aligned}
$$


Proof. This is a consequence of the following formulae, which follow from Proposition 3.5

$$
\begin{aligned}
(\Delta R i c)^{\prime} & =\Delta(R i c)^{\prime}-\frac{R}{n} \Delta h \\
\left(\nabla^{2} R\right)^{\prime} & =\nabla^{2}\left(\operatorname{tr}_{g} R i c^{\prime}\right)-\frac{R}{n} \nabla^{2}\left(\operatorname{tr}_{g} h\right), \\
((\Delta R) g)^{\prime} & =\left(\Delta\left(t r_{g} R i c^{\prime}\right)-\frac{R}{n} \Delta\left(t r_{g} h\right)\right) g, \\
\left(|R i c|^{2} g\right)^{\prime} & =\left(-2 \frac{R^{2}}{n^{2}} \operatorname{tr}_{g} h+2 \frac{R}{n} t r_{g}\left(R i c^{\prime}\right)\right) g+\frac{R^{2}}{n} h, \\
\left(R_{p k q l} R^{k l}\right)^{\prime} & =\frac{R}{n}\left(R i c_{p q}\right)^{\prime}-\frac{R}{n} R_{p k q l} h^{k l}+R_{p k q l} g^{k i} g^{l j}\left(R i c^{\prime}\right)_{i j} .
\end{aligned}
$$

Next, we linearize the Euler-Lagrange equations of $\mathcal{S}=\int R^{2}$ :

Theorem 3.7. If $g$ is Einstein, then

$$
\begin{aligned}
(\nabla \mathcal{S})^{\prime} & =2\left(\nabla^{2}\left(\operatorname{tr} R i c^{\prime}\right)-\frac{R}{n} \nabla^{2}(\operatorname{trh})\right)-2\left(\Delta\left(\operatorname{tr} R i c^{\prime}\right)-\frac{R}{n} \Delta(\operatorname{trh})\right) g \\
& -2\left(\frac{R}{n} R^{\prime} g+R R i c^{\prime}\right)+\frac{1}{2}\left(2 R R^{\prime} g+R^{2} h\right) .
\end{aligned}
$$

Proof. This is a consequence of the formulae in the proof of Theorem [3.6, together with the following:

$$
\begin{aligned}
(R \cdot R i c)^{\prime} & =\frac{R}{n}\left(R^{\prime}\right) g+R(R i c)^{\prime} \\
\left(R^{2} g\right)^{\prime} & =2 R R^{\prime} g+R^{2} h .
\end{aligned}
$$

To understand the variational properties of $\tilde{\mathcal{F}}_{\tau}$ we will rely on a splitting formula which reduces the problem to checking two types of variations: those which are traceless and divergence-free (transverse-traceless), and those which are pure trace (conformal). Therefore, we need to compute the second variation (i.e., the Jacobi operator) on each type.

3.3. Transverse-traceless variations. In this section, we assume that the variation $h$ satisfies $\delta h=0$ and $t r_{g} h=0$. Define

$$
\left(\Delta_{L} h\right)_{i j}=\Delta h_{i j}+2 R_{i p j q} h^{p q}-\frac{2}{n} R h_{i j},
$$

and note that

$$
\operatorname{Ric}^{\prime} h=-\frac{1}{2} \Delta_{L} h
$$


Theorem 3.8. In dimension $n$, if $g$ is Einstein and $h$ is transverse-traceless, then the linearized Euler-Lagrange equation of $\tilde{\mathcal{F}}_{0}$ is

$$
\left(\nabla \tilde{\mathcal{F}}_{0}\right)^{\prime} h=\frac{1}{2} \Delta_{L}^{2} h+\frac{3}{n} R \Delta_{L} h+\frac{4}{n^{2}} R^{2} h .
$$

Proof. If $h$ is transverse-traceless, then (3.19) simplifies to

$$
\begin{aligned}
\left(\nabla \mathcal{F}_{0}\right)_{p q}^{\prime} & =-\Delta(R i c)_{p q}^{\prime}+\frac{R}{n} \Delta h_{p q} \\
& -2\left(\frac{R}{n}\left(R i c_{p q}\right)^{\prime}-\frac{R}{n} R_{p k q l} h^{k l}+R_{p k q l} g^{k i} g^{l j}\left(R i c^{\prime}\right)_{i j}\right)+\frac{R^{2}}{2 n} h_{p q} .
\end{aligned}
$$

We collect terms as follows

$$
\left(-\Delta(R i c)_{p q}^{\prime}-2 R_{p k q l} g^{k i} g^{l j}\left(R i c^{\prime}\right)_{i j}\right)+\frac{R}{n}\left(\Delta h_{p q}-2\left(R i c_{p q}\right)^{\prime}+2 R_{p k q l} h^{k l}\right)+\frac{R^{2}}{2 n} h_{p q}
$$

The first term is

$$
-\Delta(R i c)_{p q}^{\prime}-2 R_{p k q l} g^{k i} g^{l j}\left(R i c^{\prime}\right)_{i j}=-\Delta_{L}(R i c)_{p q}^{\prime}-\frac{2}{n} R\left(R i c^{\prime}\right)_{p q} .
$$

Using this, the entire expression is rewritten as

$$
-\Delta_{L}(R i c)_{p q}^{\prime}+\frac{R}{n}\left(\Delta h_{p q}-4\left(R i c_{p q}\right)^{\prime}+2 R_{p k q l} h^{k l}\right)+\frac{R^{2}}{2 n} h_{p q}
$$

In the second term we have the expression

$$
\begin{aligned}
\Delta h_{p q}-4\left(R i c_{p q}\right)^{\prime}+2 R_{p k q l} h^{k l} & =\Delta h_{p q}+2 R_{p k q l} h^{k l}-4 R i c_{p q}^{\prime} \\
& =\Delta_{L} h_{p q}+\frac{2}{n} R h_{p q}+2 \Delta_{L} h_{p q}=3 \Delta_{L} h_{p q}+\frac{2}{n} R h_{p q} .
\end{aligned}
$$

Converting $R i c^{\prime}$ into $\Delta_{L}$, we obtain

$$
\frac{1}{2} \Delta_{L}^{2} h_{p q}+\frac{3}{n} R \Delta_{L} h_{p q}+\frac{n+4}{2 n^{2}} R^{2} h_{p q}
$$

Finally, for the normalized functional, we must add to (3.36) the correction term from Proposition 3.3, $-c h$, with the constant $c$ determined in Proposition 2.1, which yields (3.30).

For $\mathcal{S}$, there is a degeneracy, which is reflected in the following theorem in which the linearized Euler-Lagrange equations restricted to transverse-traceless are seen to be second order (instead of the expected fourth order).

Theorem 3.9. In dimension $n$, if $g$ is Einstein and $h$ is transverse-traceless, then the linearized Euler-Lagrange equation of $\tilde{\mathcal{S}}$ is

$$
(\nabla \tilde{\mathcal{S}})^{\prime} h=R \Delta_{L} h+\frac{2}{n} R^{2} h
$$


Proof. The only terms which will contribute anything from Theorem 3.7 are

$$
(-2 R R i c)^{\prime}=R \Delta_{L} h
$$

and the term

$$
\frac{1}{2}\left(R^{2} g\right)^{\prime}=\frac{1}{2} R^{2} h
$$

so that we obtain

$$
(\nabla \mathcal{S})^{\prime} h=R \Delta_{L} h+\frac{1}{2} R^{2} h .
$$

For the normalized functional, we must add to (3.40) the correction term from Proposition 3.3, $-c h$, with the constant $c$ determined in Proposition 2.2, which yields (3.37).

Combining Theorems 3.8 and 3.9, we obtain a useful factorization of the linearized Euler-Lagrange equations.

Theorem 3.10. In dimension $n$, if $g$ is Einstein, and $h$ is transverse-traceless, then the linearized Euler-Lagrange equation of $\tilde{\mathcal{F}}_{\tau}$ is

$$
\left(\nabla \tilde{\mathcal{F}}_{\tau}\right)^{\prime} h=\frac{1}{2}\left(\Delta_{L}+\frac{2}{n} R\right)\left(\Delta_{L}+\left(\frac{4}{n}+2 \tau\right) R\right) h .
$$

Remark 3.11. Notice that for $\tau=-(1 / n)$, the operator is a square,

$$
\frac{1}{2}\left(\Delta_{L}+\frac{2}{n} R\right)^{2} h
$$

This is not a surprise, since the functional in this case is $\int|E|^{2}$, and the kernel consists exactly of the infinitesimal Einstein deformations, i.e., transverse-traceless tensors satisfying $E^{\prime} h=0$.

3.4. Conformal Variations. Next, we consider conformal variations of the functionals $\tilde{\mathcal{F}}_{0}$ and $\tilde{\mathcal{S}}$ at an Einstein metric.

Theorem 3.12. If $h=f g$, and $g$ is Einstein, then the trace of the Jacobi operator of $\tilde{\mathcal{F}}_{0}$ is given by

$$
\operatorname{tr}(J f)=\operatorname{tr}[J(f g)]=\frac{n(n-1)}{2} \Delta^{2} f-\frac{n^{2}-10 n+8}{2 n} R \Delta f-\frac{n-4}{n} R^{2} f .
$$

Remark 3.13. As we will see below, we only need a formula for the trace of $J$, since we will be viewing $J$ as a bilinear form restricted to variations of the form $h=f g$, and

$$
\langle J(f g), f g\rangle_{L^{2}}=\langle\operatorname{tr}(J f), f\rangle_{L^{2}} .
$$

Proof. If $h=f g$, then we have

$$
\begin{aligned}
\operatorname{Ric}^{\prime} & =-\frac{1}{2}\left(\Delta f g+(n-2) \nabla_{i} \nabla_{j} f\right) \\
\operatorname{tr}\left(R i c^{\prime}\right) & =(1-n) \Delta f .
\end{aligned}
$$


Using Theorem 3.6, we compute

$$
\operatorname{tr}\left(\nabla \mathcal{F}_{0}\right)^{\prime}(f g)=\frac{n(n-1)}{2} \Delta^{2} f-\frac{n^{2}-10 n+8}{2 n} R \Delta f-\frac{n-4}{2 n} R^{2} f .
$$

For the normalized functional, from Proposition 3.3 and Proposition 2.1, we must add the term

$$
-c f=-\frac{n-4}{2 n} f
$$

and the result follows.

The analogous result for $\tilde{\mathcal{S}}$ is the following.

Theorem 3.14. If $h=f g$, and $g$ is Einstein, then the trace of the Jacobi operator of $\tilde{\mathcal{S}}$ is given by

$$
\operatorname{tr}(J f)=2(n-1)^{2} \Delta^{2} f-(n-6)(n-1) R \Delta f-(n-4) R^{2} f .
$$

Proof. In addition to (3.44) and (3.45), we note the formula

$$
R^{\prime}=(1-n) \Delta f-R f .
$$

Using Theorem 3.7, we compute

$$
\operatorname{tr}\left[\mathcal{S}^{\prime}(f g)\right]=2(n-1)^{2} \Delta^{2} f-R(n-6)(n-1) \Delta f+\frac{4-n}{2} R^{2} f .
$$

Restricting to $\mathcal{M}_{1}$, from Propositions 3.3 and 2.1, we must add the term

$$
-\operatorname{ctr} h=-\frac{n-4}{2 n} \operatorname{tr} h=-\frac{n-4}{2 n} f,
$$

and the result follows.

Combining Theorems 3.12 and 3.14, we obtain a useful factorization of the Jacobi operator.

Theorem 3.15. If $h=f g$, and $g$ is Einstein, then the trace of the Jacobi operator of $\tilde{\mathcal{F}}_{\tau}$ is given by

$$
\operatorname{tr}(J f)=\frac{1}{2 n}((n-1) \Delta+R)(n(n-4 \tau+4 n \tau) \Delta-2(n-4)(1+n \tau) R) f .
$$

\section{LOCAL VARIATIONAL PROPERTIES OF EINSTEIN METRICS}

In this section we apply the formulas of the preceding section to determine the variational properties of $\tilde{\mathcal{F}}_{\tau}$ near Einstein metrics. As in Section 3 , we consider transverse-traceless and conformal deformations separately. 
4.1. Transverse-traceless variations. We begin with the positive scalar curvature case.

Theorem 4.1. Let $(M, g)$ be an n-dimensional Einstein manifold with $R>0$.

(i) If $\tau>-1 / n$ and

$$
\operatorname{spec}_{T T}\left(-\Delta_{L}\right) \cap\left[\frac{2}{n} R,\left(\frac{4}{n}+2 \tau\right) R\right]=\varnothing,
$$

then the Jacobi operator of $\tilde{\mathcal{F}}_{\tau}$ restricted to transverse-traceless symmetric tensors is positive-definite:

$$
\int\langle J h, h\rangle d V=\int\left\langle\left(\nabla \tilde{\mathcal{F}}_{\tau}\right)^{\prime} h, h\right\rangle d V \geq \epsilon_{0} \int|h|^{2} d V
$$

for some $\epsilon_{0}>0$ and all transverse-traceless $h$.

(ii) If $\tau<-1 / n$ and

$$
\operatorname{spec}_{T T}\left(-\Delta_{L}\right) \cap\left[\left(\frac{4}{n}+2 \tau\right) R, \frac{2}{n} R\right]=\varnothing,
$$

then the same result holds.

Proof. Let $h$ be an eigensection of $\left(-\Delta_{L}\right)$ with eigenvalue $\mu_{L}$. Since $(M, g)$ is Einstein, by [Lic61] $\Delta_{L}$ maps the space of transverse-traceless $(T T)$ tensors to itself; hence we may assume $h$ is $T T$.

From (3.41), we must consider the polynomial

$$
\frac{1}{2}\left(-\mu_{L}+\frac{2}{n} R\right)\left(-\mu_{L}+\left(\frac{4}{n}+2 \tau\right) R\right) h .
$$

It is then obvious if $\mu_{L}$ is outside of the stated range, then this is strictly positive.

A similar argument (with the signs simply reversed) gives

Theorem 4.2. Let $(M, g)$ be an $n$-dimensional Einstein manifold with $R<0$.

(i) If $\tau>-1 / n$ and

$$
\operatorname{spec}_{T T}\left(-\Delta_{L}\right) \cap\left[\left(\frac{4}{n}+2 \tau\right) R, \frac{2}{n} R\right]=\varnothing,
$$

then (4.2) holds for some $\epsilon_{0}>0$ and all transverse-traceless $h$.

(ii) If $\tau<-1 / n$ and

$$
\operatorname{spec}_{T T}\left(-\Delta_{L}\right) \cap\left[\frac{2}{n} R,\left(\frac{4}{n}+2 \tau\right) R\right]=\varnothing,
$$

then the same result holds.

4.2. Conformal variations. We will let $\mathcal{M}_{1}([g])$ denote the space of unit volume metrics conformal to $g$. The tangent space of $\mathcal{M}_{1}([g])$ consists of functions with mean value zero.

For the general quadratic functional $\tilde{\mathcal{F}}_{\tau}$ we have 
Theorem 4.3. Let $(M, g)$ be an Einstein manifold of dimension $n>2$ with $R>0$. (i) Let $n=3$. If $\tau>-3 / 8$, then $g$ is a local minimizer of $\tilde{\mathcal{F}}_{\tau}$ restricted to $\mathcal{M}_{1}([g])$. If $\tau<-5 / 12$, then $g$ is a local maximizer.

(ii) Let $n=4$. If $\tau>-1 / 3$, then $g$ is a local minimizer $\tilde{\mathcal{F}}_{\tau}$ restricted to $\mathcal{M}_{1}([g])$. If $\tau<-1 / 3$, then $g$ is a local maximizer. If $\tau=-1 / 3$, then $\tilde{\mathcal{F}}_{\tau}$ is conformally invariant.

(iii) Let $n \geq 5$. If $\tau>\frac{4-3 n}{2 n(n-1)}$, then $g$ is a local minimizer of $\tilde{\mathcal{F}}_{\tau}$ restricted to $\mathcal{M}_{1}([g])$. If $\tau<-\frac{n}{4(n-1)}$, then $g$ is a local maximizer.

Furthermore, all of the above extrema are strict if $g$ is not isometric to the round sphere.

Proof. Since we are restricting to $\mathcal{M}_{1}([g])$, it suffices to prove that the second variation of the functional on functions with mean value zero is strictly positive (or strictly negative in the maximizing case).

Consider the factorization of the Jacobi operator given in 3.52. Let $\lambda$ denote a non-zero eigenvalue of $(-\Delta)$, and consider the polynomial

$$
p_{\tau}(\lambda)=\frac{1}{2 n}((n-1) \lambda-R)(n(n-4 \tau+4 n \tau) \lambda+2(n-4)(1+n \tau) R) .
$$

The eigenvalue estimate of Lichnerowicz says that

$$
\lambda_{1} \geq R /(n-1),
$$

with strict inequality if $g$ is not isometric to $S^{n}$ [Aub98, Theorem 4.19]. Consequently, the sign of $p_{\tau}$ is determined by the second factor: the functional will be minimizing, for example, if

$$
n(n-4 \tau+4 n \tau) \lambda+2(n-4)(1+n \tau) R>0 .
$$

Note that if

$$
\tau>-\frac{n}{4(n-1)} \equiv \tau_{2}
$$

then the coefficient of $\lambda$ in (4.8) is positive, so we can use the Lichnerowicz estimate again to find

$$
n(n-4 \tau+4 n \tau) \lambda+2(n-4)(1+n \tau) R \geq\left(\frac{n(n-4 \tau+4 n \tau)}{n-1}+2(n-4)(1+n \tau)\right) R .
$$

Some algebra shows that this is positive for

$$
\tau>\frac{4-3 n}{2 n(n-1)} \equiv \tau_{1}
$$

When $n=3$, then $\tau_{2}=-3 / 8>\tau_{1}=-5 / 12$, so the second factor in (4.8) is positive provided $\tau>-3 / 8$. If $n=4$, then $\tau_{2}=\tau_{1}=-1 / 3$, so the second factor is positive for $\tau>-1 / 3$. When $n \geq 5$, then $\tau_{1}>\tau_{2}$ and the second factor is positive for $\tau>\tau_{1}$. If

$$
\tau<-\frac{n}{4(n-1)}=\tau_{2},
$$


then the sign of the coefficient in of $\lambda$ in (4.8) is reversed, and we find that the second factor is negative provided

$$
\tau<\frac{4-3 n}{2 n(n-1)}=\tau_{1}
$$

If $n=3$, it follows that the second factor is negative for $\tau<\tau_{1}=-5 / 12<\tau_{2}$; when $n=4$, the second factor is negative for $\tau<-1 / 3$. When $n \geq 5$, then as $\tau_{2}<\tau_{1}$, we get negativity for $\tau<\tau_{2}$.

In the negative case, because we lack an estimate for the first eigenvalue in terms of the scalar curvature, stability often depends on an estimate of $\lambda_{1}$ :

Theorem 4.4. Let $(M, g)$ be an Einstein manifold of dimension $n>2$ with $R<0$. (i) Let $n=3$. If $\tau>-1 / 3$, then $g$ is a (strict) local minimizer of $\tilde{\mathcal{F}}_{\tau}$ restricted to $\mathcal{M}_{1}([g])$. If $\tau<-3 / 8$, then $g$ is a (strict) local maximizer.

(ii) Let $n=4$. If $\tau>-1 / 3$, then $g$ is a strict local minimizer $\tilde{\mathcal{F}}_{\tau}$ restricted to $\mathcal{M}_{1}([g])$, and is a strict local maximizer if $\tau<-1 / 3$.

(iii) Let $n \geq 5$.

- If $\tau>-1 / n$, then $g$ is a strict local minimizer of $\tilde{\mathcal{F}}_{\tau}$ restricted to $\mathcal{M}_{1}([g])$ provided

$$
\lambda_{1}>\frac{(n-4)}{2(n-1)}(-R),
$$

where $\lambda_{1}=\lambda_{1}(-\Delta)$.

- If $-\frac{n}{4(n-1)}<\tau<-1 / n$, then $g$ is a strict local minimizer of $\tilde{\mathcal{F}}_{\tau}$ restricted to $\mathcal{M}_{1}([g])$.

- If $\tau<-\frac{n}{4(n-1)}$, then $g$ is a strict local maximizer of $\tilde{\mathcal{F}}_{\tau}$ restricted to $\mathcal{M}_{1}([g])$ provided (4.13) holds.

Proof. For the same reasons as above, we restrict to the space of functions with mean value zero. For $R<0$ the first term in the factorization of $p_{\tau}$ in (4.7) is always positive, so once again the extremizing properties of the functional are determined by the sign of the second term

$$
q(\lambda)=n(n-4 \tau+4 n \tau) \lambda+2(n-4)(1+n \tau) R .
$$

First, consider the case $n \geq 5$. If $\tau>-1 / n>-\frac{n}{4(n-1)}$, then the coefficient of $\lambda$ in (4.14) is positive, and $q(\lambda)>0$ provided

$$
\lambda>\frac{2(n-4)(1+n \tau)}{n(n-4 \tau+4 n \tau)}(-R) .
$$

Note the expression on the right-hand side is increasing as a function of $\tau$, and tends (as $\tau \rightarrow \infty$ ) to $\frac{(n-4)}{2(n-1)}(-R)$. Therefore, if (4.13) holds, then $q>0$ and $g$ is a (strict) local minimizer. If $-\frac{n}{4(n-1)}<\tau<-1 / n$, then the coefficient of $\lambda$ in (4.14) is still positive, and (4.15) always holds (since the right-hand side is negative); i.e., $g$ is a local minimizer. 
If $\tau<-\frac{n}{4(n-1)}$, then the coefficient of $\lambda$ in (4.14) is negative, and $q(\lambda)<0$ provided (4.15) holds. Hence, in this case $g$ is a (strict) local maximizer. For the cases $n=3$ and $n=4$, we argue as we did in the proof of Theorem 4.3 .

The analogous results for $\tilde{\mathcal{S}}$ hold by formally setting $\tau=\infty$ in the above arguments.

Proposition 4.5. If the dimension $n \geq 3$, then an Einstein metric with $R>0$ is a local minimizer of $\tilde{\mathcal{S}}$ restricted to $\mathcal{M}_{1}([g])$, and is a strict local minimizer provided that $g$ is not isometric to $S^{n}$.

If the dimension $n=3$ or 4 , then an Einstein metric with $R<0$ is a (strict) local minimizer of $\tilde{\mathcal{S}}$ restricted to $\mathcal{M}_{1}([g])$.

If $n \geq 5$ then an Einstein metric with $R<0$ is a (strict) local minimizer of $\tilde{\mathcal{S}}$ restricted to $\mathcal{M}_{1}([g])$ provided

$$
\lambda_{1}>\frac{(n-4)}{2(n-1)}(-R) .
$$

Proof. The details are similar to above, the proof is omitted.

\section{Rigidity FOR EINSTEIN METRICS}

In this section we study the rigidity of Einstein metrics as critical points of $\tilde{\mathcal{F}}_{\tau}$. Recall by Lemma 2.12,

$$
H_{\tau}^{1}=\left\{h \in \bar{S}_{0}^{2}\left(T^{*} M\right) \mid\left(\nabla \tilde{\mathcal{F}}_{\tau}\right)_{g}^{\prime} h=0, \beta_{g} h=0\right\} .
$$

As a preliminary result we give conditions which imply that elements of $H_{\tau}^{1}$ are transverse-traceless:

Lemma 5.1. Let $(M, g)$ be an $n$-dimensional Einstein manifold which is different from the round sphere. Assume $\tau \neq-\frac{n}{4(n-1)}$.

If

$$
\frac{2(n-4)(1+n \tau)}{\left(-n^{2}+4 n \tau-4 n^{2} \tau\right)} R \notin \operatorname{spec}(-\Delta)
$$

then every $h \in H_{\tau}^{1}$ is transverse-traceless.

Remark 5.2. For the round sphere, if $\psi_{1}$ is a first-order spherical harmonic, then $h=\psi_{1} g$ is in $H_{\tau}^{1}$. However, these deformations are not essential, as they arise from paths of conformal diffeomorphisms. These can be dealt with by a modified "slicing" procedure, see Lemma 6.3 below.

Proof. Let $z$ denote the trace-free part of $h$ and write

$$
h=z+f g,
$$

where $f=\frac{1}{n}(\operatorname{tr} h)$. Note that $\beta_{g} h=0$ implies that $z$ is transverse-traceless.

We first claim that

$$
\operatorname{tr}(J h)=0 \Rightarrow \operatorname{tr}(J f)=0,
$$


where $J=\left(\nabla \tilde{\mathcal{F}}_{\tau}\right)^{\prime}$ denotes the Jacobi operator. To see this, let $\psi \in C^{\infty}(M)$. Since $J h=0$,

$$
\begin{aligned}
0 & =\langle\operatorname{tr}(J h), \psi\rangle_{L^{2}}=\langle J h, \psi \cdot g\rangle_{L^{2}}=\langle J z+J(f g), \psi g\rangle_{L^{2}} \\
& =\langle J z, \psi g\rangle_{L^{2}}+\langle J(f g), \psi g\rangle_{L^{2}}=\langle\operatorname{tr}(J z), \psi\rangle_{L^{2}}+\langle\operatorname{tr}(J f), \psi\rangle_{L^{2}} .
\end{aligned}
$$

One can check (see Theorem 3.10) that $\operatorname{tr}(J z)=0$, since $z$ is trace-free. Therefore,

$$
0=\langle\operatorname{tr}(J f), \psi\rangle_{L^{2}}
$$

for each $\psi \in C^{\infty}(M)$, which proves the claim. Consequently, if $J h=0$ then by (3.52) we have

$$
0=\operatorname{tr}(J f)=\frac{1}{2 n}((n-1) \Delta+R)(n(n-4 \tau+4 n \tau) \Delta-2(n-4)(1+n \tau) R) f .
$$

Since $(M, g)$ is not the round sphere, the first operator in the above factorization has trivial kernel. If $\tau \neq-\frac{n}{4(n-1)}$ and (5.1) holds, then the second operator also has trivial kernel, and we conclude $f=0$.

Proposition 5.3. With the same assumptions as Lemma 5.1, if we assume in addition that

$$
\left\{\frac{2}{n} R,\left(\frac{4}{n}+2 \tau\right) R\right\} \notin \operatorname{spec}_{T T}\left(-\Delta_{L}\right)
$$

then $H_{\tau}^{1}=0$.

Proof. This follows directly from Lemma 5.1 and the formula (3.41).

Therefore, to verify rigidity we need need to check two spectral conditions: one for the Laplacian on functions, the other for the Lichnerowicz Laplacian on transversetraceless tensors. The following is a summary of when the condition on $\operatorname{spec}(-\Delta)$ holds:

Lemma 5.4. Let $(M, g)$ be an Einstein manifold. Assume one of the following holds:

(i) $R>0, g$ is not round, and either

- $n=3$ and $\tau \notin(-5 / 12,-3 / 8)$;

- $n=4$ and $\tau \neq-1 / 3$; or

- $n \geq 5$ and

$$
\tau \notin\left(-\frac{n}{4(n-1)}, \frac{4-3 n}{2 n(n-1)}\right) .
$$

(ii) $g$ is Ricci-flat, and $\tau \neq-\frac{n}{4(n-1)}$.

(iii) $R<0$, and either

- $n=3$ and $\tau \notin(-3 / 8,-1 / 3)$;

- $n=4$ and $\tau \neq-1 / 3$; or

- $n \geq 5$ and

$$
\tau \in\left(-\frac{n}{4(n-1)},-\frac{1}{n}\right) .
$$

Then (5.1) holds. 
Proof. This follows immediately from the arguments in Section 4.2, where we considered the conformal Jacobi operator.

Summarizing the preceding results,

Theorem 5.5. Let $(M, g)$ be an $n$-dimensional Einstein manifold with

$$
\left\{\frac{2}{n} R,\left(\frac{4}{n}+2 \tau\right) R\right\} \notin \operatorname{spec}_{T T}\left(-\Delta_{L}\right) .
$$

If any of the cases appearing in Lemma 5.4 holds, then $H_{\tau}^{1}=0$. If $n=4$ and $\tau=-1 / 3$, then under the assumption (5.5), $H_{-1 / 3}^{1}=\{0\}$.

Proof. This follows from the above, with the added remark that in the case of $n=4$ and $\tau=-1 / 3$ (the functional $\tilde{\mathcal{W}}$ ), the space $H_{-1 / 3}^{1}$ by definition contains only TT elements, so only the condition on $\operatorname{spec}_{T T}$ is needed.

\section{Stability FOR CRITICAL METRICS}

In this section we consider stability properties of critical metrics, and give the proof of Theorems 1.4, 1.6, and 1.13. First, we recall the definition of stability:

Definition 6.1. A metric $g$ is called strictly stable if there is an $\epsilon_{0}>0$ such that the Jacobi operator $J$ associated to $\tilde{\mathcal{F}}_{\tau}$ satisfies

$$
\int\langle J h, h\rangle d V \geq \epsilon_{0} \int|h|^{2} d V
$$

for $h \in \mathcal{V} \equiv\left\{h \in C^{2, \alpha}\left(S^{2}\left(T^{*} M\right)\right) \mid \beta_{g} h=0\right\}$.

If all kernel elements are integrable and (6.1) holds for some $\epsilon_{0}>0$ and all $h \in \mathcal{V}$ orthogonal to the kernel, then $g$ is called stable.

Note that in the following proposition, we do not assume $g$ is Einstein.

Proposition 6.2. Let $(M, g)$ be critical for $\tilde{\mathcal{F}}_{\tau}$. If $g$ is stable, then $g$ is a local minimizer for $\tilde{\mathcal{F}}_{\tau}$. If $g$ is strictly stable, then $g$ is a strict local minimizer for $\tilde{\mathcal{F}}_{\tau}$.

Proof. The proof begins with a modified "slicing" lemma. Assume $\operatorname{dim}\left(H_{\tau}^{1}\right)=d>0$, and choose an $L^{2}$-orthonormal basis $\left\{u_{1}, \ldots, u_{d}\right\}$ for $H_{\tau}^{1}$. Under the assumption that all kernel elements are integrable, there is a $d$-dimensional family of critical metrics $g(Q)$ for $Q \in \mathcal{B}=B(0, \epsilon) \subset \mathbb{R}^{d}$, depending smoothly on $Q$, such that

$\left.\frac{\partial}{\partial Q_{i}} g(Q)\right|_{Q=0}=u_{i}$ for $i=1 \ldots d$, and $g(0)=g$. The notation $|Q|$ will denote the Euclidean norm of $Q$.

Lemma 6.3. For each metric $g_{1}=g+\theta_{1}$ in a sufficiently small $C^{\ell+1, \alpha}$-neighborhood of $g(\ell \geq 1)$, there exists a $C^{\ell+2, \alpha}$-diffeomorphism $\phi: M \rightarrow M$, a point $Q \in \mathbb{R}^{d}$, and a constant $c \in \mathbb{R}$ such that

$$
e^{c} \phi^{*} g_{1}=g(Q)+\tilde{\theta}
$$

with

$$
\beta_{g} \tilde{\theta}=0
$$


and

$$
\begin{gathered}
\int \operatorname{tr}_{g} \tilde{\theta} d V=0, \\
\int\left\langle u_{\nu}, \tilde{\theta}\right\rangle d V=0, \quad 1 \leq \nu \leq d .
\end{gathered}
$$

Moreover, we have the estimates

$$
\begin{gathered}
|Q| \leq C\left\|\theta_{1}\right\|_{C^{\ell+1, \alpha}}, \\
\|\tilde{\theta}\|_{C^{\ell+1, \alpha}} \leq C\left\|\theta_{1}\right\|_{C^{\ell+1, \alpha}},
\end{gathered}
$$

where $C=C(g)$.

Proof. Let $\left\{\omega_{1}, \ldots, \omega_{\kappa}\right\}$ denote a basis of the space of conformal Killing forms with respect to $g$. Define the map

$$
\mathcal{H}: C^{\ell+2, \alpha}(T M) \times \mathbb{R} \times \mathbb{R}^{d} \times \mathbb{R}^{\kappa} \times C^{\ell+1, \alpha}\left(S^{2}\left(T^{*} M\right)\right) \rightarrow C^{\ell, \alpha}\left(T^{*} M\right) \times \mathbb{R} \times \mathbb{R}^{d}
$$

by

$$
\begin{aligned}
& \mathcal{H}(X, r, Q, v, \theta)=\mathcal{H}_{\theta}(X, r, Q, v) \\
& \quad=\left(\beta_{g} \Upsilon(X, r, Q)+\sum_{i} v_{i} \omega_{i}, \int t r_{g} \Upsilon(X, r, Q) d V, \int\left\langle u_{\nu}, \Upsilon(X, r, Q)\right\rangle d V\right),
\end{aligned}
$$

where

$$
\Upsilon(X, r, Q)=e^{r} \phi_{X, 1}^{*}(g+\theta)-g(Q),
$$

and the final entry in (6.8) is understood to be an $d$-tuple. If we endow the Euclidean factors in the domain of $\mathcal{H}$ with the Euclidean norm, it is clear that $\mathcal{H}$ is a continuously differentiable mapping.

To compute the linearization at $(X, r, Q, v, \theta)=(0,0,0,0,0)$, first note that

$$
\begin{aligned}
\Upsilon^{\prime}(Y, s, P) & =\left.\frac{d}{d \epsilon} \Upsilon(\epsilon Y, \epsilon s, \epsilon P)\right|_{\epsilon=0} \\
& =s g+\mathcal{L}_{g}[Y]-\sum_{m} p_{m} u_{m} .
\end{aligned}
$$

Therefore,

$$
\begin{aligned}
\mathcal{H}_{0}^{\prime}(Y, s, P, a)= & \left(\square_{\mathcal{K}} Y^{b}-\beta_{g}\left(\sum p_{m} u_{m}\right)+\sum_{i} a_{i} \omega_{i}, n \operatorname{Vol}(g) s,\right. \\
& \left.\int\left\langle u_{\nu}, \mathcal{L}_{g} Y\right\rangle d V-\int\left\langle u_{\nu},\left(\sum_{m} p_{m} u_{m}\right)\right\rangle d V\right) \\
= & \left(\square_{\mathcal{K}} Y^{b}+\sum_{i} a_{i} \omega_{i}, n \operatorname{Vol}(g) s, \frac{2}{n} \int\left(\operatorname{tr} u_{\nu}\right)\left(\delta_{g} Y\right) d V-p_{\nu}\right) .
\end{aligned}
$$

Here we have used the fact that

$$
\beta_{g}\left(u_{\nu}\right)=0, \quad \int\left(\operatorname{tr} u_{\nu}\right) d V=0
$$


It follows that the adjoint operator is given by

$$
\left(\mathcal{H}_{0}^{\prime}\right)^{*}\left(\eta, r, q_{\mu}\right)=\left(\square_{\mathcal{K}} \eta-\frac{2}{n} \sum_{m} q_{m} d\left(\operatorname{tr} u_{m}\right), n \operatorname{Vol}(g) r,-q_{\mu}, \int\left\langle\eta, \omega_{i}\right\rangle d V\right) .
$$

Setting $\left(\mathcal{H}_{0}^{\prime}\right)^{*}\left(\eta, r, q_{\mu}\right)=0$ we immediately find (by looking at the middle two arguments) that $r=0$ and $q_{\mu}=0,1 \leq \mu \leq d$. Therefore, the cokernel condition becomes

$$
\begin{aligned}
\square_{\mathcal{K} \eta} & =0, \\
\int\left\langle\eta, \omega_{i}\right\rangle d V & =0, \quad 1 \leq i \leq \kappa .
\end{aligned}
$$

The first equation implies that $\eta$ is a conformal Killing field, while the second implies that $\eta$ is orthogonal (in $L^{2}$ ) to the space of conformal Killing forms. It follows that $\eta=0$, so the map $\mathcal{H}_{0}^{\prime}$ is surjective. Therefore, if $\theta_{1}$ is small we can find $(X, c, Q, v)$ small with

$$
\mathcal{H}_{\theta_{1}}(X, c, Q, v)=(0,0,0)
$$

In particular,

$$
\beta_{g} \Upsilon(X, c, Q)+\sum_{i} v_{i} \omega_{i}=0
$$

Fix $1 \leq \ell \leq \kappa$; if we pair both sides of (6.13) with $\omega_{\ell}$ in $L^{2}$ we find $v_{\ell}=0$, hence

$$
\beta_{g} \Upsilon(X, c, Q)=0 .
$$

Consequently, if we define $\tilde{\theta}$ by

$$
\tilde{\theta}=\Upsilon(X, c, Q)=e^{c} \phi^{*}\left(g+\theta_{1}\right)-g(Q),
$$

where $\phi=\phi_{X, 1}$, then $\tilde{\theta}$ clearly satisfies the conclusions of the Lemma.

Before we continue the proof of Proposition 6.2, we remark that there is an equivalent way of formulating the stability condition (6.1). In place of (6.1), we could require that for $h \in \mathcal{V}$.

$$
\int\langle J h, h\rangle d V \geq \epsilon_{1}\|h\|_{H^{2}}^{2}
$$

where

$$
\|h\|_{H^{2}}^{2}=\int\left|\nabla^{2} h\right|^{2} d V+\int|\nabla h|^{2} d V+\int|h|^{2} d V .
$$

We refer to (6.15) as $H^{2}$-stability. Clearly, $H^{2}$-stability implies stability. However, the converse is also true: Since $J$ is an elliptic operator on $\mathcal{V}$, we can use the spectral decomposition to conclude (6.15) from (6.1). 
Now suppose $(M, g)$ is critical for $\tilde{\mathcal{F}}_{\tau}$ and let $g_{1}=g+\theta_{1}$ be a metric which is sufficiently close in the $C^{2, \alpha}$-topology. Let $\tilde{\theta}$ satisfy (6.2)-(6.4). In particular,

$$
\begin{aligned}
\tilde{\mathcal{F}}_{\tau}[g(Q)+\tilde{\theta}] & =\tilde{\mathcal{F}}_{\tau}\left[e^{c} \phi^{*} g_{1}\right]=\tilde{\mathcal{F}}_{\tau}\left[\phi^{*} g_{1}\right] \quad \text { (by scale-invariance) } \\
& =\tilde{\mathcal{F}}_{\tau}\left[g_{1}\right]=\tilde{\mathcal{F}}_{\tau}\left[g+\theta_{1}\right] \quad \text { (by diffeomorphism-invariance). }
\end{aligned}
$$

Define

$$
a(s)=\tilde{\mathcal{F}}_{\tau}\left[g(Q)+s \frac{\tilde{\theta}}{\|\tilde{\theta}\|_{H^{2}}}\right],
$$

where $\|\tilde{\theta}\|_{H^{2}}=\|\tilde{\theta}\|_{H^{2}(g)}$. Then

$$
a(0)=\tilde{\mathcal{F}}_{\tau}[g(Q)]=\tilde{\mathcal{F}}_{\tau}[g], \quad a\left(\|\tilde{\theta}\|_{H^{2}}\right)=\tilde{\mathcal{F}}_{\tau}[g(Q)+\tilde{\theta}]=\tilde{\mathcal{F}}_{\tau}\left[g+\theta_{1}\right],
$$

and $a^{\prime}(0)=0$ since $g(Q)$ is critical. Also,

$$
\begin{aligned}
a^{\prime \prime}(0) & =\int\left\langle J_{g(Q)}\left(\frac{\tilde{\theta}}{\|\tilde{\theta}\|_{H^{2}}}\right), \frac{\tilde{\theta}}{\|\tilde{\theta}\|_{H^{2}}}\right\rangle d V_{g(Q)} \\
& =\frac{1}{\|\tilde{\theta}\|_{H^{2}}^{2}} \int\left\langle J_{g(Q)} \tilde{\theta}, \tilde{\theta}\right\rangle d V_{g(Q)} .
\end{aligned}
$$

Using the formulas for the expansion of the curvature from Section 2.4 and the fact that the second variation is only second order in the metric (see Remark 3.4), we can estimate

$$
\int\left\langle J_{g(Q)} \tilde{\theta}, \tilde{\theta}\right\rangle d V_{g(Q)}=\int\left\langle J_{g} \tilde{\theta}, \tilde{\theta}\right\rangle d V_{g}+\{\text { Error }\}
$$

where

$$
\begin{aligned}
\mid\{\text { Error }\} \mid & \leq C(g)\left[\|g(Q)-g\|_{C^{2}}+\|g(Q)-g\|_{C^{2}}^{2}\right]\|\tilde{\theta}\|_{H^{2}}^{2} \\
& \leq C\left(|Q|+|Q|^{2}\right)\|\tilde{\theta}\|_{H^{2}}^{2},
\end{aligned}
$$

since $Q \mapsto g(Q)$ is smooth. By (6.5) , if $\left\|\theta_{1}\right\|_{C^{2, \alpha}}$ is sufficiently small, then

$$
\mid\{\text { Error }\} \mid \leq C\left\|\theta_{1}\right\|_{C^{2, \alpha}} \cdot\|\tilde{\theta}\|_{H^{2}}^{2}
$$

Therefore,

$$
\begin{aligned}
a^{\prime \prime}(0) & =\frac{1}{\|\tilde{\theta}\|_{H^{2}}^{2}} \int\left\langle J_{g(Q)} \tilde{\theta}, \tilde{\theta}\right\rangle d V_{g(Q)} \\
& =\frac{1}{\|\tilde{\theta}\|_{H^{2}}^{2}}\left\{\int\left\langle J_{g} \tilde{\theta}, \tilde{\theta}\right\rangle d V_{g}+O\left(\left\|\theta_{1}\right\|_{C^{2, \alpha}}\right)\|\tilde{\theta}\|_{H^{2}}^{2}\right\} \\
& =\frac{1}{\|\tilde{\theta}\|_{H^{2}}^{2}} \int\left\langle J_{g} \tilde{\theta}, \tilde{\theta}\right\rangle d V_{g}+O\left(\left\|\theta_{1}\right\|_{C^{2, \alpha}}\right) .
\end{aligned}
$$


Also,

$$
\begin{gathered}
\int \operatorname{tr} \tilde{\theta} d V=0, \\
\int\left\langle u_{\nu}, \tilde{\theta}\right\rangle d V=0, \quad 1 \leq \nu \leq d .
\end{gathered}
$$

Since $g$ is stable, hence $H^{2}$-stable, (6.22) implies

$$
\frac{1}{\|\tilde{\theta}\|_{H^{2}}^{2}} \int\left\langle J_{g} \tilde{\theta}, \tilde{\theta}\right\rangle d V_{g} \geq \epsilon_{0}>0 .
$$

Therefore, if $\left\|\theta_{1}\right\|_{C^{2, \alpha}}$ is sufficiently small, then

$$
a^{\prime \prime}(0) \geq \epsilon_{0} / 2 \text {. }
$$

Consequently, $a(s)>a(0)$ for all $s>0$ sufficiently small (depending on $\epsilon_{0}$ ). Therefore, if $\|\tilde{\theta}\|_{C^{2, \alpha}}$ is sufficiently small (which is guaranteed, via (6.6), if $\left\|\theta_{1}\right\|_{C^{2, \alpha}}$ is small enough), then

$$
\tilde{\mathcal{F}}_{\tau}[g]=\tilde{\mathcal{F}}_{\tau}[g(Q)]<\tilde{\mathcal{F}}_{\tau}[g(Q)+\tilde{\theta}]=\tilde{\mathcal{F}}_{\tau}\left[g+\theta_{1}\right] .
$$

In particular, if $\tilde{F}_{\tau}\left[g+\theta_{1}\right]=\tilde{\mathcal{F}}_{\tau}[g]$ then $\tilde{\theta}=0$. It follows that $g$ is a local minimum of $\tilde{\mathcal{F}}_{\tau}$.

If $g$ is strictly stable, then $\operatorname{dim}\left(H_{\tau}^{1}\right)=0$ and in place of Lemma 6.3 we can simply apply the slicing result of Lemma 2.10 , and the rest of the proof goes through to show that $g$ is a strict local minimum of $\tilde{\mathcal{F}}_{\tau}$.

Combining the results of Theorems 4.1 and 4.3 with the preceding proposition, we can now prove the main stability result, Theorem 1.4 of the Introduction:

Proof of Theorem 1.4. Suppose (1.8) holds. By Theorems 4.1 and 4.3, the Jacobi operator $J=J_{g}$ of $\tilde{\mathcal{F}}_{\tau}$ is positive definite when restricted to either transverse-traceless or conformal variations. Let $h$ satisfy $\beta_{g} h=0$; we need to show

$$
\int\langle J h, h\rangle d V \geq \epsilon_{0} \int|h|^{2} d V
$$

for some $\epsilon_{0}>0$. Write $h=z+f g$, where $z$ is the trace-free part of $h$; then

$$
0=\beta_{g} h=\beta_{g} z+\beta_{g}(f g)=\delta_{g} z
$$

Therefore, $z$ is transverse-traceless. Then

$$
\int\langle J h, h\rangle d V=\int\langle J z, z\rangle d V+\int\langle J(f g), f g\rangle d V+2 \int\langle J z, f g\rangle d V,
$$


where the last line follows from the fact that $J$ is self-adjoint (so we can combine the cross-terms). However, the cross term vanishes because $\operatorname{tr}(J z)=0$ for $z$ transversetraceless (as easily seen by taking a trace of (3.41)). Therefore,

$$
\begin{aligned}
\int\langle J h, h\rangle d V & =\int\langle J z, z\rangle d V+\int\langle J(f g), f g\rangle d V \\
& \geq \epsilon_{0} \int\left[|z|^{2}+f^{2}\right] d V \geq \epsilon_{0} \int|h|^{2} d V
\end{aligned}
$$

We conclude that $g$ is strictly stable, so Theorem 1.4 follows from Proposition 6.2 ,

In the case of the Bach tensor, using the terminology introduced in Definition 1.5, we have the following analogue to Proposition 6.2.

Proposition 6.4. Let $\left(M^{4}, g\right)$ be Bach-flat. If $g$ is (strictly) Bach-stable, then $g$ is a (strict) local minimizer for $\mathcal{W}$.

Proof. The details are similar to the proof of Proposition 6.2, with the following modification. We introduce an extra conformal factor as in the slicing in Theorem 2.11, to arrange moreover that $\operatorname{tr}_{g_{0}}(\tilde{\theta})=0$ in Lemma 6.3. Since the functional is conformally invariant, the argument in the proof of Proposition 6.2 then extends to this case.

Next, we prove the main theorem regarding Bach-flat metrics.

Proof of Theorem 1.6. The rigidity statement follows from Theorem 5.5 and Corollary 2.14. For the local minimization statement, strict Bach-stability follows from Theorems 4.1 and 4.2 , and the local minimization statement then follows from Proposition 6.4.

6.1. Applications to reverse Bishop's inequalities. One consequence of stability is a reverse Bishop's inequality, Theorem 1.13 of the Introduction:

Proof of Theorem 1.13. Since $g$ is stable, for all metrics $\tilde{g}$ in a $C^{2, \alpha}$-neighborhood of $g$ we have

$$
\begin{aligned}
\tilde{\mathcal{F}}_{0}[\tilde{g}] & =\operatorname{Vol}(\tilde{g})^{4 / n-1} \int|\operatorname{Ric}(\tilde{g})|^{2} d V_{\tilde{g}} \\
& \geq \tilde{\mathcal{F}}_{0}[g]=\operatorname{Vol}(g)^{4 / n-1} \int|\operatorname{Ric}(g)|^{2} d V_{g}=n(n-1)^{2} \operatorname{Vol}(g)^{4 / n},
\end{aligned}
$$

and equality holds if and only if $\tilde{g}$ is (up to scaling) isometric to $g$. Assume $\tilde{g}$ satisfies

$$
\operatorname{Ric}(\tilde{g}) \leq(n-1) \tilde{g} \text {. }
$$

An additional condition on the neighborhood $U$ is that $\tilde{g}$ also satisfies

$$
\operatorname{Ric}(\tilde{g})>-(n-1) \tilde{g}
$$

and consequently

$$
|\operatorname{Ric}(\tilde{g})|^{2} \leq n(n-1)^{2},
$$


hence

$$
\tilde{\mathcal{F}}_{0}[\tilde{g}]=\operatorname{Vol}(\tilde{g})^{4 / n-1} \int|\operatorname{Ric}(\tilde{g})|^{2} d V \leq n(n-1)^{2} \operatorname{Vol}(\tilde{g})^{4 / n} .
$$

Combining this with (6.23) we get

$$
\operatorname{Vol}(\tilde{g}) \geq \operatorname{Vol}(g) .
$$

Moreover, if equality holds then $\tilde{g}=e^{c} \phi^{*} g$, but since $g$ and $\tilde{g}$ have the same volume, $c=0$. This completes the proof.

A similar argument gives

Corollary 6.5. Let $(M, g)$ be a negative Einstein manifold, normalized so that

$$
\operatorname{Ric}(g)=-(n-1) g \text {. }
$$

Assume

$$
\lambda_{1}\left(-\Delta_{L}\right)=\lambda_{L}>\frac{2}{n} R
$$

(i) If $n=3$ or 4 , then there exists a $C^{2, \alpha}$-neighborhood $U$ of $g$ such that if $\tilde{g} \in U$ with

$$
\operatorname{Ric}(\tilde{g}) \geq-(n-1) \tilde{g},
$$

then

$$
\operatorname{Vol}(\tilde{g}) \geq \operatorname{Vol}(g)
$$

and equality holds if and only if $\tilde{g}$ is isometric to $g$.

(ii) If $n \geq 5$, assume in addition

$$
\lambda_{1}(-\Delta)>\frac{2(n-4)(n-1)}{n}
$$

Then the same conclusion holds.

\section{EXAMPLES}

In this section, we apply the above computations to various examples and determine conditions for rigidity and stability.

\subsection{The round sphere $\left(S^{n}, g_{S}\right)$ (or any quotient thereof).}

Proof of Theorem 1.7. Normalizing so that $\operatorname{Ric}\left(g_{s}\right)=(n-1) g_{S}$, the Lichnerowicz Laplacian on TT-tensors is easily verified to be

$$
\Delta_{L} h=\Delta h-2 n h .
$$

The inequality

$$
\int_{S^{n}}\left|\nabla_{i} h_{j k}+\nabla_{j} h_{k i}+\nabla_{k} h_{i j}\right|^{2} d V \geq 0
$$


implies that the least eigenvalue of the rough Laplacian on TT-tensors is $2 n$ [Koi78]. Consequently, least eigenvalue of the Lichnerowicz Laplacian on TT-tensors is

$$
4 n=\frac{4}{n-1} R \text {. }
$$

Theorem 4.1 then implies that if

$$
\tau<\frac{2}{n(n-1)}
$$

then the Jacobi operator is positive definite when restricted to TT-tensors. For the conformal direction, we can simply quote Theorem 4.3 to obtain the lower restriction on $\tau$. If $(M, g)$ is a proper quotient of $S^{n}$, then the theorem follows from Proposition 6.2. In the case of the round metric, for $\tau$ in the specified range, $H_{\tau}^{1}$ consists of pure trace elements of the form $\{f g: f \in \Lambda\}$, where $\Lambda$ is the space of first-order spherical harmonics. These directions are clearly integrable, since they arise from conformal diffeomorphisms. Local minimization then follows from Proposition 6.2. However, since all of the minimizing metrics are isometric, we actually have strict local minimization.

The final statement in the theorem regarding $\tilde{\mathcal{R}}$ follows since $\tau=-1 /(2(n-1))$ is always included in the above ranges of $\tau$, and since these metrics obviously minimize $\tilde{\mathcal{W}}$ (they are locally conformally flat), together with (1.13). form.

As a consequence of $\tau=0$, we see that Theorem 1.13 applies to any spherical space

Remark 7.1. We next briefly discuss the size of the neighborhood $U$ appearing in the above theorem. Given $\epsilon>0$ small and a constant $\Lambda$ large, there exists a $\delta>0$ such that if

$$
\|R m-(1 / 2) g \otimes g\|_{C^{0}}<\delta,\|R m\|_{C^{\alpha}}<\Lambda,
$$

then $\left\|g-g_{S}\right\|_{C^{2, \alpha}}<\epsilon$ where $g_{S}$ is a constant curvature metric [Pet06, Chapter 10]. In the case of a space form, we suspect that the neighborhood of stability in Theorem 1.4 can be weakened from pointwise $C^{2, \alpha}$-norm to the integral condition

$$
\|R m(\tilde{g})-(1 / 2) \tilde{g} \otimes \tilde{g}\|_{L^{n / 2}}<\delta
$$

for $\delta$ sufficiently small.

We next consider the rigidity of space forms.

Theorem 7.2. Let $(M, g)$ be a spherical space form, and let $\tau$ be in the ranges specified in Lemma 5.4 .

(i) If $(M, g)$ is not isometric to the sphere, then $H_{\tau}^{1}$ contains only traceless tensors.

(ii) On $\left(S^{n}, g_{S}\right)$, the pure trace elements in $H_{\tau}^{1}$ are exactly $\{f g: f \in \Lambda\}$, where $\Lambda$ is the space of first-order spherical harmonics.

In either case, if one orders the TT-spectrum of $\left(-\Delta_{L}\right)$ as

$$
0<\mu_{1}=4 n<\mu_{2}<\mu_{3}<\cdots,
$$


then $H_{\tau}^{1}$ contains non-zero TT-tensors exactly at the sequence of discrete values

$$
\tau_{i}=\frac{1}{2 n(n-1)}\left(\mu_{i}-4(n-1)\right) .
$$

If $n=4$ and $\tau=-1 / 3$, then $H_{\tau}^{1}=\{0\}$, thus any spherical space form is Bach-rigid.

Proof. This easily follows from Theorem 5.5 and Corollary 2.14.

As seen above in the proof Theorem 1.7, on the round sphere, for $\tau$ such that $H_{\tau}^{1}=\{f g: f \in \Lambda\}, g_{S}$ is indeed rigid, since these directions are tangent to conformal diffeomorphisms, and the moduli in these directions are all isometric.

Proof of Theorem 1.8. As in [Lam98], we consider the Lie group $S U(2)$, and choose the following basis of the Lie algebra $\mathfrak{s u}(2)$

$$
\left\{\left(\begin{array}{cc}
i & 0 \\
0 & -i
\end{array}\right),\left(\begin{array}{cc}
0 & i \\
i & 0
\end{array}\right),\left(\begin{array}{cc}
0 & -1 \\
1 & 0
\end{array}\right)\right\}
$$

Let $\left\{e_{1}, e_{2}, e_{3}\right\}$ denote the corresponding basis of left-invariant vector fields. We declare these to be orthogonal with $e_{1}$ and $e_{2}$ of length 1 , and $e_{3}$ of length $s$ for positive $s \in \mathbf{R}$. The resulting metric is known as a Berger sphere, and for $s=1$, is the round metric $g_{S}$. It follows that $\left\{\tilde{e}_{1}, \tilde{e}_{2}, \tilde{e}_{3}\right\}=\left\{e_{1}, e_{2}, \frac{1}{s} e_{3}\right\}$ is an orthonormal frame satisfying the commutation relations

$$
\left[\tilde{e}_{1}, \tilde{e}_{2}\right]=2 s \tilde{e}_{3}, \quad\left[\tilde{e}_{2}, \tilde{e}_{3}\right]=\frac{2}{s} \tilde{e}_{1}, \quad\left[\tilde{e}_{3}, \tilde{e}_{1}\right]=\frac{2}{s} \tilde{e}_{2} .
$$

From these relations, it easily follows that in this basis the Ricci tensor is diagonal with eigenvalues $\left\{4-2 s^{2}, 4-2 s^{2}, 2 s^{2}\right\}$. We then have

$$
\begin{gathered}
|R i c|^{2}(s)=32-32 s^{2}+12 s^{4}, \\
R^{2}(s)=64-32 s^{2}+4 s^{4},
\end{gathered}
$$

and therefore

$$
|R i c|^{2}+\tau R^{2}=32(1+2 \tau)-32(1+\tau) s^{2}+4(3+\tau) s^{4} .
$$

Consequently,

$$
\tilde{\mathcal{F}}_{\tau}\left[g_{s}\right]=c \tau^{4 / 3}\left(32(1+2 \tau)-32(1+\tau) s^{2}+4(3+\tau) s^{4}\right),
$$

for some constant $c>0$. At the value of $\tau=1 / 3$, it is easily verified that

$$
\left.\frac{d \tilde{\mathcal{F}}_{\tau}\left[g_{s}\right]}{d s}\right|_{s=1}=0 \text {, and }\left.\frac{d^{2} \tilde{\mathcal{F}}_{\tau}\left[g_{s}\right]}{d s^{2}}\right|_{s=1}=0 \text {, but }\left.\frac{d^{3} \tilde{\mathcal{F}}_{\tau}\left[g_{s}\right]}{d s^{3}}\right|_{s=1}>0
$$

which shows that $s=1$ is not a strict minimizer, and therefore $\tau=1 / 3$ is sharp for stability

Finally, $h=g_{s}^{\prime}$ is a multiple of $e_{3} \otimes e_{3}$, so modulo a constant multiple of the metric, is in the lowest eigenspace of $\left(-\Delta_{L}\right)$, which is $H_{1 / 3}^{1}$. It is straightforward to see that if there were any path of unit-volume critical metrics $\tilde{g}_{s}$ with $\tilde{g}_{s}^{\prime}=h$, then (7.8) would hold for $\tilde{g}_{s}$. This is a contradiction since the functional would be constant along $\tilde{g}_{s}$. 
Remark 7.3. For $-1 / 2<\tau<-1 / 3$, it is easy to see that that there is another critical metric for some value $s<1$, which is a local maximum in the Berger-parameter direction. As in [Lam98, these are examples of non-Einstein critical metrics.

\subsection{Hyperbolic manifolds.}

Proof of Theorem 1.9. Let $(M, g)$ be a compact hyperbolic manifold in dimension $n$, satisfying $\operatorname{Ric}(g)=-(n-1) g$. The Lichnerowicz Laplacian on TT-tensors is

$$
\Delta_{L} h=\Delta h+2 n h .
$$

The inequality

$$
\int_{M}\left|\nabla_{i} h_{j k}-\nabla_{j} h_{i k}\right|^{2} d V \geq 0
$$

implies that the least eigenvalue of the rough Laplacian on TT-tensors is $n$, with equality for Codazzi tensors Koi78. Consequently, on a hyperbolic manifold, the least eigenvalue of the rough Laplacian on TT-tensors is bounded below by

$$
-n=\frac{1}{n-1} R
$$

Theorem 4.2 implies that $\tilde{\mathcal{F}}_{\tau}$ is strictly stable in the TT-direction for

$$
\tau>\frac{4-3 n}{2 n(n-1)} .
$$

Combining this with Theorem 4.4 for strict stability in the conformal direction, this proves strict stability. Strict local minimization then follows from Proposition 6.2. The local minimization statement for $\tilde{\mathcal{R}}$ for $n=3$ and $n=4$ follows as in the spherical case.

Remark 7.4. For $n \geq 5$, the upper endpoint may be improved given an improved estimate on the lowest eigenvalue of the Laplacian on functions. It is easy to show that if

$$
\lambda_{1}>\frac{2(n-4)}{n^{2}}(-R),
$$

then the interval may be extended to include $\tau=0$.

We next consider the case of rigidity of compact hyperbolic manifolds.

Theorem 7.5. Let $(M, g)$ be a compact hyperbolic manifold, and let $\tau$ be in the ranges specified in Lemma 5.4. Then $H_{\tau}^{1}$ contains no pure-trace elements. If one orders the TT-spectrum of $\left(-\Delta_{L}\right)$ as

$$
-n \leq \mu_{1}<\mu_{2}<\mu_{3}<\cdots,
$$

then $H_{\tau}^{1}$ contains non-zero TT-tensors only at the sequence of discrete values

$$
\tau_{i}=-\frac{1}{2 n(n-1)}\left(\mu_{i}+4(n-1)\right) \text {. }
$$

Proof. This easily follows from Theorem 5.5. 
Remark 7.6. In the case of $n=4, \tau=-1 / 3$, a non-trivial TT-deformation yields equality in (7.9), which means that $h$ is a Codazzi tensor. Such deformations are exactly locally conformally flat deformations, and in this situation the hyperbolic structure is called bendable [Laf83, JM87].

\subsection{Complex projective space $\mathbb{C P}^{m}$.}

Proof of Theorem 1.10. We begin with an eigenvalue estimate on $\mathbb{C P}^{m}$ proved using representation theory in the case $m=2$ in [War82, and for all $m$ by direct calculation in Bou10a].

Proposition 7.7 ([Bou10a, War82]). On $\left(\mathbb{C P}^{m}, g_{F S}\right)$, with Ric $=2(m+1) g$, the least eigenvalue of the Lichnerowicz Laplacian on TT-tensors is

$$
8(m+2)=\frac{2(m+2)}{m(m+1)} R .
$$

Theorem 4.1 then implies that if

$$
\tau<\frac{1}{m(m+1)}
$$

then $\tilde{\mathcal{F}}_{\tau}$ is strictly stable in the TT-direction. For the conformal direction, we can simply quote Theorem 4.3. This proves strict stability, and strict local minimization then follows from Proposition 6.2. The statement for $\mathcal{R}$ in dimension 4 follows for the reasons as discussed in the introduction.

As a consequence of $\tau=0$, we see that Theorem 1.13 applies to to $\left(\mathbb{C P}^{m}, g_{F S}\right)$.

We next consider the case of rigidity in the case of $m=2$.

Theorem 7.8. On $\mathbb{C P}^{2}$, the Fubini-Study metric $g_{F S}$ is infinitesimally rigid (and thus rigid) provided that $\tau<1 / 6$. If one orders the TT-spectrum of $\left(-\Delta_{L}\right)$ as

$$
0<\mu_{1}=32<\mu_{2}<\mu_{3}<\cdots,
$$

then $H_{\tau}^{1}$ is nontrivial only at the sequence of discrete values

$$
\tau_{i}=\frac{1}{48}\left(\mu_{i}-24\right) .
$$

For $\tau=-1 / 3, H_{\tau}^{1}=0$, thus $g_{F S}$ is Bach-rigid.

Proof. This easily follows from Theorem 5.5 and Corollary 2.14.

As in the case of the sphere, we conjecture that the infinitesimal deformations at the value $\tau_{i}$ are not integrable.

7.4. The product metric on $S^{m} \times S^{m}$. In this subsection, we will discuss the proof of Theorem 1.11.

Proposition 7.9. On $\left(S^{m} \times S^{m}, g_{1}+g_{2}\right)$, with Ric $=(m-1) g$, the least eigenvalue of the Lichnerowicz Laplacian on TT-tensors is 0, with 1-dimensional kernel spanned by $g_{1}-g_{2}$. The next eigenvalue is greater than or equal to $2 \mathrm{~m}$. 
Proof. As in [Kob85, the general traceless symmetric tensor splits as

$$
h=\stackrel{\circ}{h_{1}}+\frac{f}{m} g_{1}+\hat{h}+\stackrel{\circ}{h_{2}}-\frac{f}{m} g_{2},
$$

where $h_{1}$ is tangent to the first factor, $\stackrel{\circ}{h}$ is its trace-free part, $h_{2}$ is tangent to the second factor, and $\hat{h}$ are the mixed directions. The above decomposition is orthogonal, and is preserved by $\Delta_{L}$. The curvature tensor is given by

$$
R_{i j k l}=\left(g_{1}\right)_{i k}\left(g_{1}\right)_{j l}-\left(g_{1}\right)_{j k}\left(g_{1}\right)_{i l}+\left(g_{2}\right)_{i k}\left(g_{2}\right)_{j l}-\left(g_{2}\right)_{j k}\left(g_{2}\right)_{i l},
$$

with Ric $=(m-1) g$, and $R=2 m(m-1)$. If $h$ satisfies $\left(-\Delta_{L}\right) h=\lambda h$, then we have the separate equations

$$
\begin{aligned}
\stackrel{\circ}{h}_{i}-2 m \stackrel{\circ}{h}_{i} & =(-\lambda) \stackrel{\circ}{h_{i}} \\
\Delta f & =(-\lambda) f \\
\Delta \hat{h}-2(m-1) \hat{h} & =(-\lambda) \hat{h} .
\end{aligned}
$$

Lemma 7.10. The lowest eigenvalue of $\left(-\Delta_{L}\right)$ acting on tensors of the form $h=h_{1}$ or $h=\stackrel{\circ}{h}_{2}$ is at least $4 m$ if $h$ is divergence-free. If $h$ is not divergence-free, then the smallest eigenvalue is at least $2(m+1)$.

Proof. Without loss of generality, assume $h=\stackrel{\circ}{h}$. We first assume that $h$ is divergencefree. Next symmetrize and consider

$$
\begin{aligned}
& \int\left|\nabla_{i} h_{j k}+\nabla_{j} h_{k i}+\nabla_{k} h_{i j}\right|^{2} d V=3 \int|\nabla h|^{2} d V+6 \int \nabla_{i} h_{j k} \nabla_{j} h_{k i} d V \\
& =3 \int|\nabla h|^{2} d V-6 \int h_{j k} \nabla_{i} \nabla_{j} h_{k i} d V \\
& =3 \int|\nabla h|^{2} d V-6(m-1) \int|h|^{2} d V+6 \int h_{j k} R_{i j k}^{p} h_{p i} d V .
\end{aligned}
$$

Thus we have the inequality

$$
\int|\nabla h|^{2} d V \geq 2(m-1) \int|h|^{2} d V-2 \int h_{j k} R_{i j k}^{p} h_{p i} d V .
$$

Since $h$ is "tangent" to the first $S^{m}$-factor and $h$ is traceless, the last integral is

$$
-2 \int h_{j k} R_{i j k}^{p} h_{p i} d V=2 \int|h|^{2} d V .
$$

Substituting this into (7.25), we obtain

$$
\int|\nabla h|^{2} d V \geq 2(m-1) \int|h|^{2} d V+2 \int|h|^{2} d V=2 m \int|h|^{2} d V .
$$

From (7.21), the least eigenvalue of $\left(-\Delta_{L}\right)$ on divergence-free is therefore at least $2 m+2 m=4 m$. 
Next if $h$ is not divergence-free, we use the formula [Lic61]

$$
\delta\left(\Delta_{L} h\right)=-\Delta_{H}(\delta h)
$$

So if $h$ is an eigenfunction of $\left(-\Delta_{L}\right)$ with eigenvalue $\nu$, then $\delta h$ is a eigenfunction of $\Delta_{H}$ with the same eigenvalue.

From Folland [Fol89], the eigenspaces of $\Delta_{H}$ on 1-forms on $S^{m}$ are spanned by the 1 -forms $\phi_{1 \ell}$ with eigenvalues $(\ell+1)(\ell+m-2)$, and $\psi_{1 \ell}$ with eigenvalues $\ell(\ell+m-1)$. Note that $\delta \phi_{1 \ell}=0$, and $d \psi_{1 \ell}=0$. The first few eigenvalues of $\phi_{1 \ell}$ are

$$
2(m-1), 3 m, 4(m+1), \ldots
$$

These are all of the divergence-free eigenforms. Note that the $2(m-1)$-eigenvalue here corresponds to Killing forms. The first few eigenvalues of $\psi_{1 \ell}$ are

$$
m, 2(m+1), 3(m+2), \ldots
$$

This list is exactly the eigenvalues of the Laplacian on functions (excepting the zero eigenvalue); this is not an accident since $\psi_{1 \ell}=d f_{\ell}$ where $f_{\ell}$ is a harmonic function with the same eigenvalue, so none of these are divergence-free.

The formula

$$
\Delta_{L} h=\Delta h-2 m h
$$

tells us that the least eigenvalue of $\left(-\Delta_{L}\right)$ is at least $2 m$, with equality if and only if $\Delta h=0$, in which case $h$ is parallel. Then $h$ is necessarily divergence-free, and the above argument says the least eigenvalue of $\Delta$ on divergence-free tensors is at least $4 m$, so this cannot happen. Consequently, the least eigenvalue is strictly greater than $2 m$.

Next, the argument in [BGM71] says that the eigenspace of the Hodge Laplacian on 1-forms with eigenvalue $\mu$ is spanned by forms of the form

$$
f_{1}\left(z_{1}\right) \alpha_{2}\left(z_{2}\right), f_{2}\left(z_{2}\right) \alpha_{1}\left(z_{1}\right),
$$

where $f_{i}$ is an eigenfunction of the Laplacian on the $i$-th $S^{m}$-factor with eigenvalue $\nu$, and $\alpha_{i}$ is an eigen-1-form of the Hodge Laplacian $\tilde{\nu}$ for $i=1,2$ with $\mu=\nu+\tilde{\nu}$. Consequently, the list of eigenvalues on 1-forms on the product is given by all sums from (7.28) and the second list (7.29) taken with the second list beginning with 0 . Therefore the list of eigenvalues on 1-forms on the product begins with

$$
m, 2(m-1), 2 m, 2(m+1), 3 m-2,3 m, \ldots
$$

We already know that $\nu>2 n$, so the next possiblity is $2(m+1)$.

The preceding lemma says that for any eigenvalue strictly less than $2(m+1)$, we must have $\stackrel{\circ}{h}=0$ for $i=1,2$. So we next consider $h$ of the form

$$
h=\frac{f}{m} g_{1}+\hat{h}-\frac{f}{m} g_{2} .
$$


In this case, we have the two equations

$$
\begin{aligned}
\Delta f & =(-\lambda) f \\
\Delta \hat{h}-2(m-1) \hat{h} & =(-\lambda) \hat{h} .
\end{aligned}
$$

We recall the eigenvalues of the Laplacian on functions on $S^{m}$ are

$$
0, m, 2(m+1), 3(m+2), \ldots
$$

The argument in [BGM71] says that the eigenspace of the Laplacian on with eigenvalue $\mu$ is spanned by functions of the form

$$
f_{1}\left(z_{1}\right) f_{2}\left(z_{2}\right),
$$

where $f_{i}$ is an eigenfunction of the Laplacian on the $i$-th factor with eigenvalue $\nu$ for $i=1,2$ with $\mu=\nu+\tilde{\nu}$. Consequently, the list of eigenvalues on functions on the product is given by all sums from the list (7.36) with itself, which begins with

$$
0, m, 2 m, 2(m+1), \ldots
$$

Lemma 7.11. For divergence-free $h$ of the form $h=\hat{h}$, we have

$$
\int|\nabla \hat{h}|^{2} \geq 2(m-1) \int|\hat{h}|^{2}
$$

Proof. As in the proof of Lemma 7.10, we symmetrize to obtain

$$
\int|\nabla h|^{2} d V \geq 2(m-1) \int|h|^{2} d V-2 \int h_{j k} R_{i j k}^{p} h_{p i} d V .
$$

Since the curvature tensor has no mixed terms, the last term vanishes, and the result follows.

Continuing with the argument, first, assume that $f$ is non-zero. The first possibility is $\lambda=0$, in which case $f$ is constant. Since $h$ is divergence-free, it follows that $\delta \hat{h}=0$, and the Proposition then implies that $\hat{h}=0$. This case does in fact happen, since $g_{1}-g_{2}$ is a kernel element.

Still assuming $f$ is non-zero, the next possibility is $\lambda=m$. In this case $f$ is either $f\left(z_{1}\right)$ or $f\left(z_{2}\right)$, where $f$ is a first eigenfunction on $S^{m}$. Without loss of generality, assume that $f=f\left(z_{1}\right)$. Then if $\hat{h}$ is non-zero, the divergence-free condition on $h$ yields

$$
\delta \hat{h}=-\frac{1}{m} d f .
$$

Integrating, we obtain

$$
\int\langle d f, d f\rangle d V=-m \int\langle d f, \delta \hat{h}\rangle d V=m \int\left\langle\nabla^{2} f, \hat{h}\right\rangle d V=0,
$$

since the hessian of $f$ only lives on the first factor, and $\hat{h}$ has only mixed components. This implies $d f=0$, which is a contradiction since $f$ is not-constant. Therefore $\hat{h}=0$, which again says $d f=0$, which is a contradiction. 
In the above 2 paragraphs, we have shown that if $f$ is non-zero, then $\lambda \geq 2 m$ unless $\lambda=0$, and $f$ is constant. So we next consider the case that $\lambda<2 m$. If $f$ is a non-zero constant, then $\lambda=0$. Then $\delta \hat{h}=0$, and Lemma 7.11 then implies that $\hat{h}=0$. This proves the zero eigenspace is spanned by $g_{1}-g_{2}$. Next, if $\lambda<2 m$ and $f$ is not constant, then $f$ must vanish. In this case, $\delta \hat{h}=0$, and the proposition says $\lambda \geq 4(m-1)$, which is a contradiction.

Therefore we have shown that the kernel of $\Delta_{L}$ on traceless divergence-free tensors is spanned by $g_{1}-g_{2}$, and that the next largest eigenvalue is at least $2 \mathrm{~m}$.

Next, we show that for $m=2$, then 4 is actually an eigenvalue. We let

$$
\hat{h}=\alpha_{1} \odot \alpha_{2},
$$

where $\alpha_{i}$ is a divergence-free eigenform of the rough Laplacian on 1-forms on the $i$-th factor, $i=1,2$. Here $\odot$ denotes symmetric product. We have seen above that the lowest eigenvalue of the rough Laplacian on $S^{2}$ on divergence-free 1 -forms is 1 . We then have

$$
\begin{aligned}
\Delta\left(\alpha_{1} \odot \alpha_{2}\right) & =\Delta\left(\alpha_{1} \otimes \alpha_{2}+\alpha_{2} \otimes \alpha_{1}\right) \\
& =\Delta\left(\alpha_{1}\right) \otimes \alpha_{2}+\alpha_{1} \otimes \Delta\left(\alpha_{2}\right)+\Delta\left(\alpha_{2}\right) \otimes \alpha_{1}+\alpha_{2} \otimes \Delta\left(\alpha_{1}\right) \\
& =-2 \alpha_{1} \otimes \alpha_{2}-2 \alpha_{2} \otimes \alpha_{1}=-2\left(\alpha_{1} \odot \alpha_{2}\right),
\end{aligned}
$$

and $\alpha_{1} \odot \alpha_{2}$ is clearly traceless and divergence-free, with (7.23) showing that the corresponding eigenvalue of $\Delta_{L}$ is 4 .

Remark 7.12. In higher dimensions, Lemma 7.11 shows that the least eigenvalue of $\left(-\Delta_{L}\right)$ on divergence-free $h$ of the form $\hat{h}$ is $4(m-1)$. This does in fact occur as an eigenvalue by considering $\alpha_{1} \odot \alpha_{2}$ as we did above for $m=2$.

Noting that

$$
2 m=\frac{1}{m-1} R
$$

Theorem 4.1 then implies that if

$$
\tau<\frac{2-m}{2 m(m-1)}
$$

then $\tilde{\mathcal{F}}_{\tau}$ is strictly stable in the TT-direction. For the conformal direction, we can simply quote Theorem 4.3. Strict local minimization then follows from Proposition 6.2, and the statement for $\mathcal{R}$ in dimension 4 follows for the reasons as discussed in the introduction. this completes the proof of Theorem 1.11.

We next consider the case of rigidity for $S^{2} \times S^{2}$.

Theorem 7.13. On $S^{2} \times S^{2}$, the product metric $g_{1}+g_{2}$ is infinitesimally rigid (and therefore rigid) provided that $\tau<-1 / 2$. If one orders the TT-spectrum of $\left(-\Delta_{L}\right)$ as

$$
\mu_{1}=0<\mu_{2}=4<\mu_{3}<\cdots,
$$


then $H_{\tau}^{1}$ is nontrivial only at the sequence of discrete values

$$
\tau_{i}=\frac{1}{8}\left(\mu_{i}-4\right)
$$

For $\tau_{1}=-(1 / 2), \operatorname{dim}\left(H_{-1 / 2}^{1}\right)=1$, and the path of metrics $s \mapsto e^{s} g_{1}+e^{-s} g_{2}$ yields a corresponding non-trivial deformation, with $h=g_{1}-g_{2}$. This is a Kähler constant scalar curvature deformation, which is a path of critical metrics for the functional $\tilde{\mathcal{F}}_{\tau_{1}}$, therefore these infinitesimal deformations are integrable.

For $\tau_{2}=0, \operatorname{dim}\left(H_{0}^{1}\right)=9$, and this space is spanned by tensors of the form $\alpha_{1} \odot \alpha_{2}$ where $\alpha_{i}$ is a Killing form on the $i$-th factor, $i=1,2$.

For $\tau=-1 / 3, H_{\tau}^{1}=0$, thus $g_{1}+g_{2}$ is Bach-rigid [Kob85].

Proof. The follows from Theorem [5.5 and Corollary 2.14. The fact that $H_{-1 / 2}^{1}$ is spanned $g_{1}-g_{2}$ follows from Proposition 7.9 . The fact that $g_{s}$ are critical for $\tilde{\mathcal{F}}_{\tau_{1}}$ follows from [Der83, Equation 38], which says that the Bach tensor of Kähler constant scalar curvature surface is a constant multiple of the traceless Ricci tensor, which is exactly the Euler-Lagrange equation for $\tau_{1}=-1 / 2$. The determination of $H_{0}^{1}$ was carried out above.

As in the previous examples, we conjecture that the infinitesimal deformations at value $\tau_{i}$ for $i \geq 2$ are not integrable.

\subsection{The Ricci-flat case.}

Proof of Theorem 1.12. Theorem 3.15 shows that the Jacobi operator on pure trace elements is

$$
\operatorname{tr}(J f)=(n-4 \tau+4 n \tau)(n-1) \Delta^{2} f .
$$

Consequently, $g$ is strictly stable in the conformal direction for $-n / 4(n-1)<\tau$.

Theorem 3.10 shows that the Jacobi operator on TT-tensors is a positive multiple of $\Delta_{L}^{2}$, and is therefore non-negative. Integrating by parts, a TT-kernel element $h$ satisfies $\Delta_{L} h=0$, and is therefore an infinitesimal Einstein deformation. Integrability of infinitesimal Einstein deformations on the torus is elementary, and the integrability of such deformations on a Calabi-Yau manifold was proved by Bogomolov and Tian [Bog78, Tia87. Theorem 1.12 then follows from Proposition 6.2.

\section{REFERENCES}

[And97] Michael T. Anderson, Extrema of curvature functionals on the space of metrics on 3manifolds, Calc. Var. Partial Differential Equations 5 (1997), no. 3, 199-269.

[And01] Extrema of curvature functionals on the space of metrics on 3-manifolds. II, Calc. Var. Partial Differential Equations 12 (2001), no. 1, 1-58.

[Aub98] Thierry Aubin, Some nonlinear problems in Riemannian geometry, Springer Monographs in Mathematics, Springer-Verlag, Berlin, 1998.

[AV12] Antonio G. Ache and Jeff A. Viaclovsky, Obstruction-flat asymptotically locally Euclidean metrics, Geom. Funct. Anal. 22 (2012), no. 4, 832-877.

[Bac21] R. Bach, Zur Weylschen Relativitatstheorie und der Weylschen Erweiterung des Krummungstensorbegriffs, Math. Zeit. 9 (1921), 112-135. 
[BCG95] G. Besson, G. Courtois, and S. Gallot, Entropies et rigidités des espaces localement symétriques de courbure strictement négative, Geom. Funct. Analysis 5 (1995), no. 5, $731-799$.

[BCG99] _ Lemme de Schwarz réel et applications géométriques, Acta Math. 183 (1999), no. $2,145-169$.

[Ber03] Marcel Berger, A panoramic view of Riemannian geometry, Springer-Verlag, Berlin, 2003.

[Bes87] Arthur L. Besse, Einstein manifolds, Ergebnisse der Mathematik und ihrer Grenzgebiete (3) [Results in Mathematics and Related Areas (3)], vol. 10, Springer-Verlag, Berlin, 1987.

[BGM71] Marcel Berger, Paul Gauduchon, and Edmond Mazet, Le spectre d'une variété riemannienne, Lecture Notes in Mathematics, Vol. 194, Springer-Verlag, Berlin, 1971.

[Bla00] David E. Blair, Spaces of metrics and curvature functionals, Handbook of differential geometry, Vol. I, North-Holland, Amsterdam, 2000, pp. 153-185.

[Bog78] F. A. Bogomolov, Hamiltonian Kählerian manifolds, Dokl. Akad. Nauk SSSR 243 (1978), no. $5,1101-1104$.

[Bou10a] Mohamed Boucetta, Spectra and symmetric eigentensors of the Lichnerowicz Laplacian on $\mathrm{P}^{n}(\mathbb{C})$, J. Geom. Phys. 60 (2010), no. 10, 1352-1369.

[Bou10b] Vincent Bour, Fourth order curvature flows and geometric applications, arXiv:1012.0342, 2010.

[CT94] Jeff Cheeger and Gang Tian, On the cone structure at infinity of Ricci flat manifolds with Euclidean volume growth and quadratic curvature decay, Invent. Math. 118 (1994), no. 3, 493-571.

[Der83] Andrzej Derdziński, Self-dual Kähler manifolds and Einstein manifolds of dimension four, Compositio Math. 49 (1983), no. 3, 405-433.

[DWW05] Xianzhe Dai, Xiaodong Wang, and Guofang Wei, On the stability of Riemannian manifold with parallel spinors, Invent. Math. 161 (2005), no. 1, 151-176.

[DWW07] _ On the variational stability of Kähler-Einstein metrics, Comm. Anal. Geom. 15 (2007), no. 4, 669-693.

[Ebi70] David G. Ebin, The manifold of Riemannian metrics, Global Analysis (Proc. Sympos. Pure Math., Vol. XV, Berkeley, Calif., 1968), Amer. Math. Soc., Providence, R.I., 1970, pp. 11-40.

[FM77] Arthur E. Fischer and Jerrold E. Marsden, The manifold of conformally equivalent metrics, Canad. J. Math. 29 (1977), no. 1, 193-209.

[Fol89] G. B. Folland, Harmonic analysis of the de Rham complex on the sphere, J. Reine Angew. Math. 398 (1989), 130-143.

[GL91] C. Robin Graham and John M. Lee, Einstein metrics with prescribed conformal infinity on the ball, Adv. Math. 87 (1991), no. 2, 186-225.

[Gur98] Matthew J. Gursky, The Weyl functional, de Rham cohomology, and Kähler-Einstein metrics, Ann. of Math. (2) 148 (1998), no. 1, 315-337.

[GV01] Matthew J. Gursky and Jeff A. Viaclovsky, A new variational characterization of threedimensional space forms, Invent. Math. 145 (2001), no. 2, 251-278.

[JM87] Dennis Johnson and John J. Millson, Deformation spaces associated to compact hyperbolic manifolds, Discrete groups in geometry and analysis (New Haven, Conn., 1984), Progr. Math., vol. 67, Birkhäuser Boston, Boston, MA, 1987, pp. 48-106.

[Kob85] Osamu Kobayashi, On a conformally invariant functional of the space of Riemannian metrics, J. Math. Soc. Japan 37 (1985), no. 3, 373-389.

[Koi78] Norihito Koiso, Nondeformability of Einstein metrics, Osaka J. Math. 15 (1978), no. 2, 419-433.

[Koi80] - Rigidity and stability of Einstein metrics — the case of compact symmetric spaces, Osaka J. Math. 17 (1980), no. 1, 51-73. 
[Koi82] _ Rigidity and infinitesimal deformability of Einstein metrics, Osaka J. Math. 19 (1982), no. 3, 643-668.

[Laf83] Jacques Lafontaine, Modules de structures conformes plates et cohomologie de groupes discrets, C. R. Acad. Sci. Paris Sér. I Math. 297 (1983), no. 13, 655-658.

[Lam98] François Lamontagne, A critical metric for the $L^{2}$-norm of the curvature tensor on $S^{3}$, Proc. Amer. Math. Soc. 126 (1998), no. 2, 589-593.

[LeB01] Claude LeBrun, Ricci curvature, minimal volumes, and Seiberg-Witten theory, Invent. Math. 145 (2001), no. 2, 279-316.

[LeB04] C Curvature functionals, optimal metrics, and the differential topology of 4manifolds, Different faces of geometry, Int. Math. Ser. (N. Y.), vol. 3, Kluwer/Plenum, New York, 2004, pp. 199-256. MR 2102997 (2005h:53055)

[Lic61] André Lichnerowicz, Propagateurs et commutateurs en relativité générale, Inst. Hautes Études Sci. Publ. Math. (1961), no. 10, 56.

[LP11] H. Lü and C. N. Pope, Critical gravity in four dimensions, Phys. Rev. Lett. 106 (2011), 181302.

[Mal11] J. Maldacena, Einstein gravity from conformal gravity, arXiv:1105.5632v2, 2011.

[Mut74] Yosio Mutō, Curvature and critical Riemannian metric, J. Math. Soc. Japan 26 (1974), 686-697.

[Pal] Richard Palais, unpublished manuscript.

[Pet06] Peter Petersen, Riemannian geometry, second ed., Graduate Texts in Mathematics, vol. 171, Springer, New York, 2006.

[RS05] Yann Rollin and Michael Singer, Non-minimal scalar-flat Kähler surfaces and parabolic stability, Invent. Math. 162 (2005), no. 2, 235-270.

[Sch07] Hans-Jürgen Schmidt, Fourth order gravity: equations, history, and applications to cosmology, Int. J. Geom. Methods Mod. Phys. 4 (2007), no. 2, 209-248.

[Smo07] N.K. Smolentsev, Spaces of Riemannian metrics, J. Math. Sci. (N. Y.) 142 (2007), no. 5, 2436-2519.

[Ste78] K. S. Stelle, Classical gravity with higher derivatives, General Relativity and Gravitation 9 (1978), no. 4, 353-371.

[Str08] Jeffrey D. Streets, The gradient flow of $\int_{M}|\mathrm{Rm}|^{2}$, J. Geom. Anal. 18 (2008), no. 1, 249-271.

[Str12] Jeffrey Streets, The gradient flow of the $L^{2}$ curvature energy near the round sphere, Adv. Math. 231 (2012), no. 1, 328-356.

[Tia87] Gang Tian, Smoothness of the universal deformation space of compact Calabi-Yau manifolds and its Petersson-Weil metric, Mathematical aspects of string theory (San Diego, Calif., 1986), Adv. Ser. Math. Phys., vol. 1, World Sci. Publishing, Singapore, 1987, pp. 629-646.

[TV05] Gang Tian and Jeff Viaclovsky, Moduli spaces of critical Riemannian metrics in dimension four, Adv. Math. 196 (2005), no. 2, 346-372.

[Via06] Jeff Viaclovsky, Conformal geometry and fully nonlinear equations, Inspired by S. S. Chern, Nankai Tracts Math., vol. 11, World Sci. Publ., Hackensack, NJ, 2006, pp. 435460 .

[War82] N. P. Warner, The spectra of operators on $\mathbf{C P}^{n}$, Proc. Roy. Soc. London Ser. A 383 (1982), no. 1784, 217-230.

[Ye93] Rugang Ye, Ricci flow, Einstein metrics and space forms, Trans. Amer. Math. Soc. 338 (1993), no. 2, 871-896.

[Yu03] Zheng Yu, The negative gradient flow for the $L^{2}$-integral of Ricci curvature, Manuscripta Math. 111 (2003), no. 2, 163-186. 
Department of Mathematics, University of Notre Dame, Notre Dame, IN 46556

E-mail address: mgursky@nd.edu

Department of Mathematics, University of Wisconsin, Madison, Wi 53706

E-mail address: jeffv@math.wisc.edu 